\title{
Spectroscopy of Rydberg states in erbium using electromagnetically induced transparency
}

\author{
A. Trautmann $\odot,{ }^{1}$ M. J. Mark $\odot,{ }^{1,2}$ P. Ilzhöfer,,${ }^{1, *}$ H. Edri, ${ }^{1}$ A. El Arrach $\odot,{ }^{1}$ J. G. Maloberti, ${ }^{1}$ \\ C. H. Greene $\odot,{ }^{3,4}$ F. Robicheaux $\odot,,^{3,4}$ and F. Ferlaino $\oplus^{1,2}$ \\ ${ }^{1}$ Institut für Quantenoptik und Quanteninformation, Österreichische Akademie der Wissenschaften, \\ Technikerstraße 21a, 6020 Innsbruck, Austria \\ ${ }^{2}$ Institut für Experimentalphysik, Universität Innsbruck, Technikerstraße 25, 6020 Innsbruck, Austria \\ ${ }^{3}$ Department of Physics and Astronomy, Purdue University, West Lafayette, Indiana 47907, USA \\ ${ }^{4}$ Purdue Quantum Science and Engineering Institute, Purdue University, West Lafayette, Indiana 47907, USA
}

(Received 3 May 2021; accepted 15 July 2021; published 19 August 2021)

\begin{abstract}
We present a study of the Rydberg spectrum in ${ }^{166} \mathrm{Er}$ for series connected to the $4 f^{12}\left({ }^{3} H_{6}\right) 6 s, J_{c}=13 / 2$, and $J_{c}=11 / 2$ ionic core states using an all-optical detection based on electromagnetically induced transparency in an effusive atomic beam. Identifying approximately 550 individual states, we find good agreement with a multichannel quantum defect theory (MQDT) which allows assignment of most states to $n s$ or $n d$ Rydberg series. We provide an improved accuracy for the lowest two ionization thresholds to $E_{\mathrm{IP}, J_{c}=13 / 2}=49260.750(1) \mathrm{cm}^{-1}$ and $E_{\mathrm{IP}, J_{c}=11 / 2}=49701.184(1) \mathrm{cm}^{-1}$ as well as the corresponding quantum defects for all observed series. We identify Rydberg states in five different isotopes, and states between the two lowest ionization thresholds. Our results open the way for future applications of Rydberg states for quantum simulation using erbium and exploiting its special open-shell structure.
\end{abstract}

DOI: 10.1103/PhysRevResearch.3.033165

\section{INTRODUCTION}

Rydberg states in neutral atoms have been highly successful in realizing strongly interacting many-body quantum platforms. The large dipole-dipole (van der Waals) interaction, typically exceeding the $\mathrm{MHz}$ scale, enables unique paths for quantum information processing and many-body quantum simulations [1-3]. The conditions of strong interaction, long internal-state coherence, and microscopic control can now be satisfied simultaneously when driving Rydberg (Ry) excitations in tweezer-trapped neutral atoms [4]. This important advance was initially developed with alkaline atoms [5-8]. Due to their relatively sparse atomic structure, alkali are well suited for the implementation of robust, yet simple, cooling and trapping methods. However, this simplicity comes at the cost of somehow restricted opportunities for state preparation and manipulation of the available internal degrees of freedom.

Recently, there has been growing interest in extending the Rydberg toolbox to more complex multi-valence-electron atomic species, opening possibilities for, e.g., laser cooling and trapping, high-fidelity optical read-out, and quantuminformation storage [9-12]. In multi-valence-electron atoms,

\footnotetext{
*Present address: 5. Physikalisches Institut and Center for Integrated Quantum Science and Technology, Universität Stuttgart, Pfaffenwaldring 57, 70569 Stuttgart, Germany.

Published by the American Physical Society under the terms of the Creative Commons Attribution 4.0 International license. Further distribution of this work must maintain attribution to the author(s) and the published article's title, journal citation, and DOI.
}

the key paradigm shift is that, after Ry excitation of one of the available valence electrons, the core remains optically active, effectively resembling a single-charged positive ion. This active core leads, for instance, to a comparatively large polarizability [11], allowing for optical trapping despite the repulsive ponderomotive potential for the Ry electron [13]. Remarkable progress has been made with two-electron atoms, i.e., the alkaline-earth $\mathrm{Sr}$ [14-17] and the alkaline-earth-like $\mathrm{Yb}$ atoms [18], for ground-state tweezer trapping [19-21], and, more recently, the combination of tweezer trapping and Ry excitation [13,22].

Pushing the boundaries even further, the next step is to consider atomic species with more than two valence electrons, like open-shell lanthanides for which laser cooling and quantum degeneracy have been demonstrated [23-27]. Compared to alkaline earth, these species might exhibit an ionic-core polarizability resembling even more the ground-state polarizability, and might allow access to a large hyperfine manifold $[9,12]$. Besides the plethora of laser cooling transitions, they could also allow direct access to high orbital-momentum Ry states with negligible quantum defects, which are expected to have strong pair interactions because of the multitude of nearby degenerate quantum states. However, being a comparatively new quantum resource, open-shell Ry lanthanides remain rather unexplored in the ultracold regime.

So far, the only reported high-resolution Ry spectroscopy in ultracold open-shell lanthanides has been performed with holmium in a magneto-optical trap (MOT) [28]. For Dy and $\mathrm{Pm}$, resonance ionization spectroscopy (RIS) has been performed on a hot atomic vapor [29-31]. Using RIS, preliminary data for erbium are also available [32].

The present paper reports on the first high-resolution Ry spectroscopy of erbium atoms. Using a two-photon scheme 

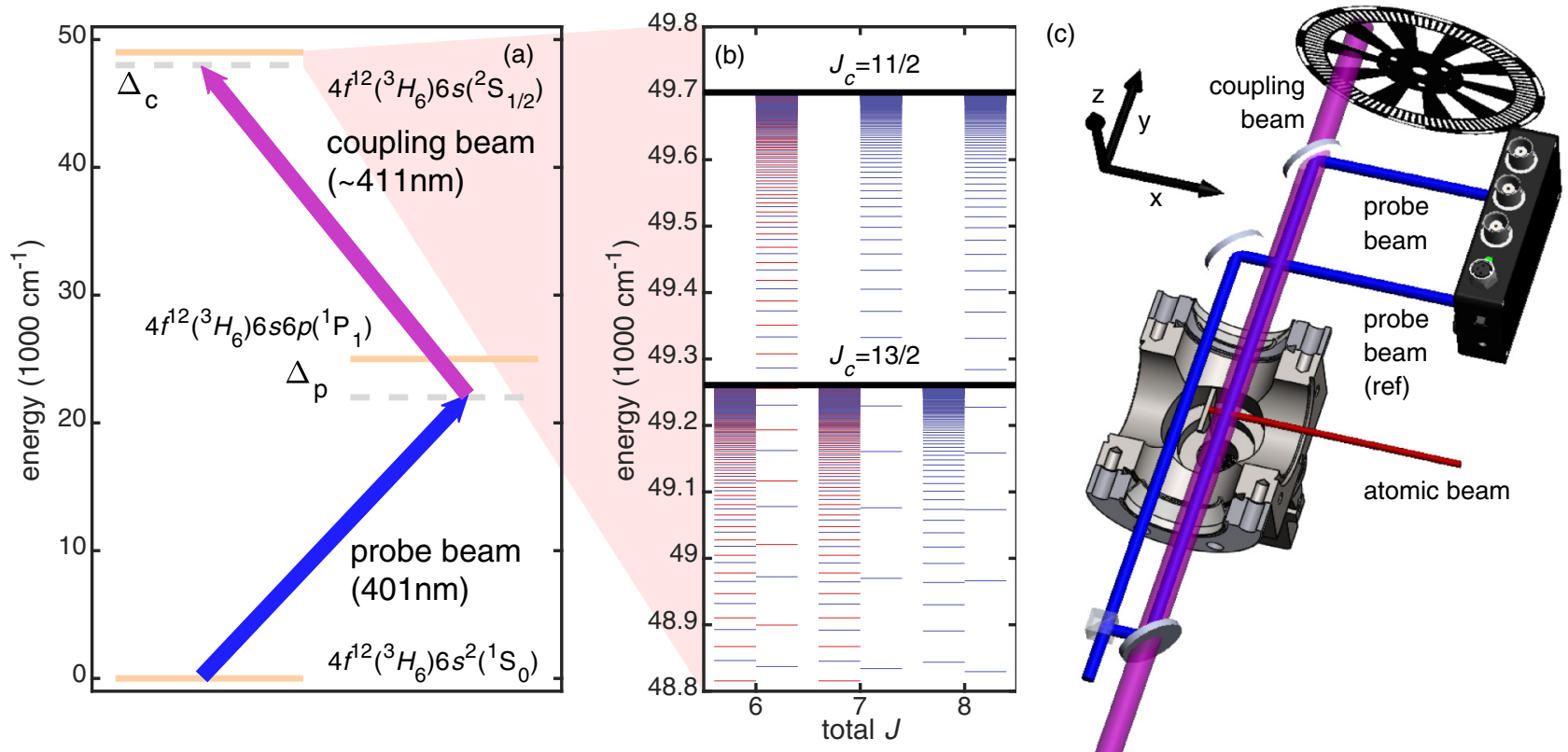

FIG. 1. Electronic levels and experimental setup. (a) Excitation scheme involving the probe transition at about $401 \mathrm{~nm}$ with single photon detuning $\Delta_{p}$ and the coupling transition around $411 \mathrm{~nm}$. Also shown, the two-photon detuning $\Delta_{c}$. (b) Schematic Rydberg level scheme of the two lowest ionization thresholds for different total $J$. Red (blue) lines indicate the $n s(n d)$ series. (c) Schematic drawing of the experimental setup with the vacuum apparatus, atomic beam, and probe, reference, and couple laser beams.

based on electromagnetically induced transparency (EIT) [33-36], we observe the $n s$ and $n d$ Rydberg series with principal quantum number $n$ ranging from 15 up to 140 . Using slope minimizing fits in Lu-Fano style plots [37], we provide an improved value of the first ionization potential. Our method does not require the magnetic quadrupole field present in a MOT, and thus allows an effortless high-resolution study of Zeeman shifts of the Rydberg states. We use these shifts to assign the total angular momentum, $J$, of a subset of Ry $n s$ and $n d$ states, which serves as an important input parameter for the modeling of the Rydberg series.

Moreover, we identify characteristics of the Rydberg states using procedures based on multichannel quantum defect theory (MQDT), similar to recent work on Sr [38] but accounting for Rydberg perturbers from a spin-orbit split threshold. Using the approximation that the $n s$ and $n d$ states do not mix at all, we successfully perform a MQDT fit to the $n s$ series. For the nd series, we introduce two different approximate methods which are fairly successful in representing most of the states but are less precise in representing the $n d$ perturbers or the measured $g$ factors.

Finally, we surprisingly observe that using just a twophoton transition we could presumably couple ground-state atoms to an $n g$ Ry state $(\Delta \ell=4)$ thanks to the interaction between the submerged shells in Lanthanides. This result provides a first example of the uniqueness of Ry lanthanide with respect to alkali and alkaline-earth atoms.

The paper is structured in the following way: Section II discusses the energy levels and coupling schemes of erbium with all relevant states for this paper. The experimental setup and measurement techniques are reviewed in Sec. III. In Sec. IV, we will discuss the experimental data by demonstrating the EIT signal and present the determination of $J$ and $g_{J}$ for a set of $n d$ states as well as an accurate value for the ionization threshold. Section V presents our MQDT results for both $n s$ and $n d$ series and will compare the results with our experimental results.

\section{CONSIDERATIONS ON THE ERBIUM LEVEL STRUCTURE}

Figure 1(a) shows the excitation scheme used in this paper and Fig. 1(b) a zoom-in on the most relevant Rydberg series attached to the lowest two ionization thresholds. The electronic ground-state configuration of erbium reads $\left[4 f^{12}\left({ }^{3} H_{6}\right) 6 s^{2}\left({ }^{1} S_{0}\right)\right]_{6}$. This type of configuration is often called submerged because the partially filled inner $f$ shell is surrounded by the outer $s$ shell. Erbium has a total of 14 valence electrons and each of those can be excited to a higher-lying state. The interactions inside the submerged shell determines the type of angular momentum coupling of the state. Generally speaking, the spin-orbit coupling scheme depends on the specific configuration and on the energy of the state-i.e., overlap between the submerged wave functions.

For our two-photon Rydberg spectroscopy, we couple the ground state to the intermediate state $\left[4 f^{12}\left({ }^{3} H_{6}\right) 6 s 6 p\left({ }^{1} \mathrm{P}_{1}\right)\right]_{7}$. Here, the angular momentum coupling results from the small size of the $4 f$ orbital compared to that of the $s p$ electrons. This leads to the $4 f^{12}$ electrons coupling together to give a particular angular momentum term, $\left({ }^{3} H_{6}\right)$, and the $s p$ electrons coupling to give $\left({ }^{1} \mathrm{P}_{1}\right)$. These two partial $J$ 's are then coupled to give the total $J=7$ for the intermediate state. A similar argument holds for the ground and first excited state of the ion $\left[4 f^{12}\left({ }^{3} H_{6}\right) 6 s\left({ }^{2} \mathrm{~S}_{1 / 2}\right)\right]_{J_{c}}$ with $J_{c}=13 / 2$ for the ground ionic state and $J_{c}=11 / 2$ for the first excited state. For the Rydberg states, the energy scale of the Rydberg electron is 
TABLE I. Quantum numbers of all relevant states for the EIT scheme, with the total ground state, intermediate state, and ion core states with Rydberg series attached. The table lists the configuration, state energy, as well as total angular momentum $J$. For Rydberg states, the core total angular momentum $J_{c}$ is also given. Only states with $|\Delta J| \leqslant 1$ with respect to the intermediate state $\left(J_{\mathrm{ex}}=7\right)$ are shown as our two-photon scheme only couples to such states.

\begin{tabular}{lcc}
\hline \hline Configuration & Term, $J$ & Energy $\left(\mathrm{cm}^{-1}\right)$ (threshold) \\
\hline $4 f^{12}\left({ }^{3} H_{6}\right) 6 s^{2}\left({ }^{1} \mathrm{~S}_{0}\right)$ & $(6,0) 6$ & 0 \\
$4 f^{12}\left({ }^{3} H_{6}\right) 6 s 6 p\left({ }^{1} \mathrm{P}_{1}\right)$ & $(6,1) 7$ & 24943.298 \\
$4 f^{12}\left({ }^{3} H_{6}\right) 6 s_{1 / 2}$ & $(6,1 / 2) 13 / 2$ & 49260.750 \\
$4 f^{12}\left({ }^{3} H_{6}\right) 6 s_{1 / 2}$ & $(6,1 / 2) 11 / 2$ & 49701.184 \\
$4 f^{12}\left({ }^{3} H_{6}\right) 6 s_{1 / 2} n s_{1 / 2}$ & $(13 / 2,1 / 2) 6,7$ & $\left(J_{c}=13 / 2\right)$ \\
$4 f^{12}\left({ }^{3} H_{6}\right) 6 s_{1 / 2} n s_{1 / 2}$ & $(11 / 2,1 / 2) 6$ & $\left(J_{c}=11 / 2\right)$ \\
$4 f^{12}\left({ }^{3} H_{6}\right) 6 s_{1 / 2} n d_{3 / 2}$ & $(13 / 2,3 / 2) 6,7,8$ & $\left(J_{c}=13 / 2\right)$ \\
$4 f^{12}\left({ }^{3} H_{6}\right) 6 s_{1 / 2} n d_{5 / 2}$ & $(13 / 2,5 / 2) 6,7,8$ & $\left(J_{c}=13 / 2\right)$ \\
$4 f^{12}\left({ }^{3} H_{6}\right) 6 s_{1 / 2} n d_{3 / 2}$ & $(11 / 2,3 / 2) 6,7$ & $\left(J_{c}=11 / 2\right)$ \\
$4 f^{12}\left({ }^{3} H_{6}\right) 6 s_{1 / 2} n d_{5 / 2}$ & $(11 / 2,5 / 2) 6,7,8$ & $\left(J_{c}=11 / 2\right)$ \\
\hline \hline
\end{tabular}

the smallest, suggesting the angular momentum of the positive ion should be coupled to that of the Rydberg electron. There are three ways to order the addition of angular momenta but the interaction between the Rydberg states will typically preclude the states from being nearly pure in one ordering or the other. The order used in the MQDT simulations is to add the spin and orbital angular momenta of the Rydberg electron together to get the total angular momentum, $j$, of the Rydberg electron; this is then added to the total angular momentum of the core electrons to get the total angular momentum of the final state. Our two-photon excitation scheme leads to even parity Rydberg states with most of the states having $n s$ or nd character. Also, Lanthanides offer the unique possibility to directly couple to $n g$ states with $\ell=4$ due to the angular momentum of the submerged shell. Table I lists the relevant quantum numbers for the states discussed in this paper.

\section{EXPERIMENTAL SETUP}

Figure 1(c) shows a schematic drawing of the experimental spectroscopy setup, consisting of an ultrahigh vacuum setup with a high-temperature effusion cell, a transversal cooling chamber, a differential pumping section (not shown here), and the probe chamber. The design is similar to the one in Ref. [39]. Erbium atoms are evaporated in the effusion cell at $1300{ }^{\circ} \mathrm{C}$. From the effusion cell, the atoms pass through three apertures to form a collimated beam, propagating along the horizontal $x$ direction. In the subsequent probe chamber, the atomic beam crosses the interaction region with the coupling and probe laser beam. The coupling and probe beam counterpropagate and intersect the atomic beam perpendicular $(y$ direction) to reduce Doppler shifts. They are overlapped and separated by dichroic mirrors. An additional reference beam, split from the probe beam, propagates parallel to the probe beam and acts as a reference to cancel out power fluctuations. We can block the atomic beam between the probe and reference beam to provide a reference with and without the atomic absorption. We use about 10 to $500 \mu \mathrm{W}$, with a waist $\left(1 / e^{2}\right.$ radius) of about $0.5 \mathrm{~mm}$ for both the probe and reference beam, while the coupling beam approximately has $130 \mathrm{~mW}$ at the interaction region and a waist of $1 \mathrm{~mm}$. We modulate the coupling beam with an optical chopper at $7 \mathrm{kHz}$, monitor both the probe and reference beam on balanced photodiodes (PD1 and PD2), and feed the ac-coupled difference of these signals as input for a lock-in amplifier. Both lasers are commercial resonantly frequency-doubled devices; the probe and reference beam are derived from an amplified diode laser locked onto the $401 \mathrm{~nm}$ transition line using a Doppler-free modulation transfer spectroscopy in a hollow cathode lamp [25]. The coupling laser is derived from a continuous-wave free-running Ti:Sa laser. Both lasers are monitored on a wavelength meter with $60 \mathrm{MHz}$ absolute accuracy, see Appendix C.

We add quarter- and half-wave plates to the probe and coupling beam path to control the light polarization, and apply a magnetic field on the order of $10 \mathrm{G}$ in the $z\left(B=\left(0,0, B_{z}\right)\right)$ direction. This enables us to drive $\sigma^{ \pm}$and/or $\pi$ transitions in a controlled setting, which facilitates the assignment of the total angular momentum $J$ and estimation of the $g$ factor of Rydberg states, see Sec. IV B.

\section{DATA ANALYSIS}

To detect the Rydberg levels, we make use of EIT [33]. In short, we detect the probe laser transmission through the atomic beam, which experiences absorption when in resonance with the atomic transition to the intermediate state. In case the coupling laser hits a resonance condition, i.e., couples the intermediate state to a Rydberg state, this absorption gets reduced. This can be understood in a dressed state picture where the coupling leads to a doublet of dressed states (Autler-Townes doublet) [40] and, together with destructive interference of the absorption of these states, to a transparency window at resonance. This tell-tale sign of EIT is shown in Fig. 2(a), where we directly record the probe laser power after passing the atomic beam. The probe laser frequency is scanned over the absorption resonance, while the coupling laser frequency is fixed, in this case on resonance to the Rydberg state at $49147.967 \mathrm{~cm}^{-1}$, identified as the lowest-lying fine-structure state of the $31 d$ Rydberg manifold, see later discussion. The narrow transmission peak due to EIT appears in the center of the absorption line.

For a survey of Rydberg states, we lock the probe laser onto the hollow-cathode lamp spectroscopy on resonance with the $401 \mathrm{~nm}$ transition to the intermediate state, scan the coupling laser frequency, and again record the transmitted probe laser power. We additionally improve our signal-to-noise ratio by using a reference beam, which we subtract to reduce noise from power fluctuations, and using a lock-in technique where we modulate the coupling beam with a chopper, see Sec. II for details. In case we hit the resonance condition to a Rydberg state, we observe directly the increased transmission. Figure 2(b) shows an excerpt of the total spectroscopy data in the region from about $49112 \mathrm{~cm}^{-1}$ to $49211 \mathrm{~cm}^{-1}$.

Using this technique, we record about 550 EIT resonances and assign their total energy, see Appendix D for a full list. After the determination of the ionization thresholds (see details in Sec. IV A) we can assign effective quantum numbers to each level. Figure 2(c) shows the energy of all 

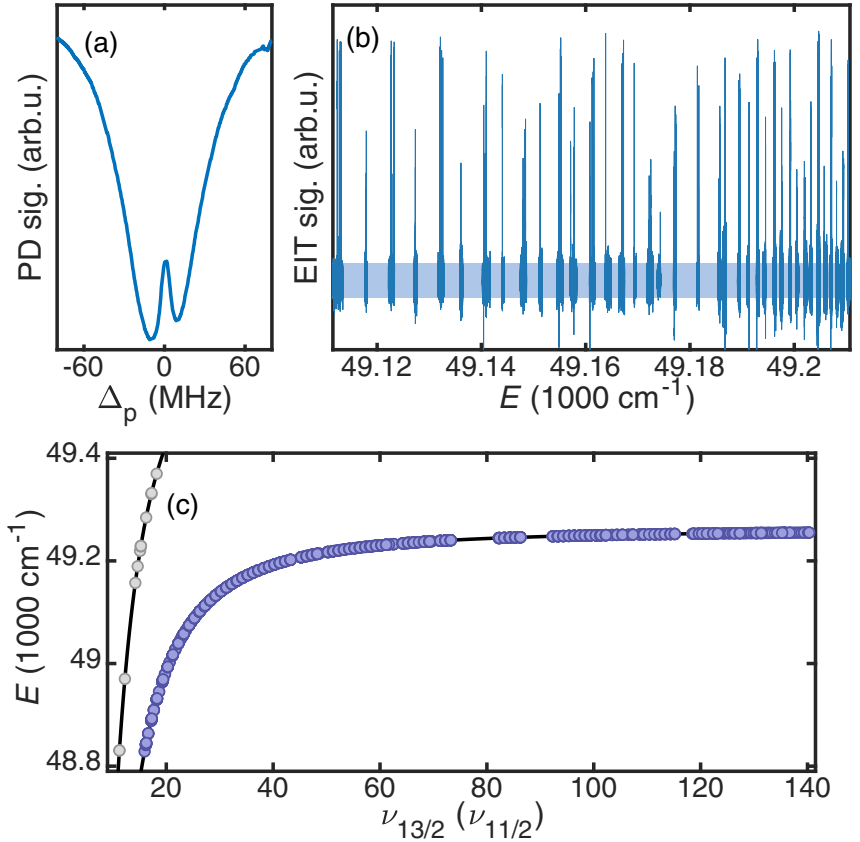

FIG. 2. EIT spectroscopy and survey of Rydberg states. (a) Exemplary EIT resonance around $49147.967 \mathrm{~cm}^{-1}$. Here, the coupling beam is kept fixed while $\Delta_{p}$ is scanned, showing the typical EIT signal of reduced absorption when on two-photon resonances. (b) EIT spectroscopy over a broad range of energies covering about 100 individual Rydberg states. $\Delta_{p}$ is fixed to zero while the coupling laser frequency is scanned. The shaded area indicates the noise floor as a guide to the eye. (c) Energy of all observed Rydberg states extracted from the data as a function of the assigned effective principle quantum number. Ry states that are either above the first ionization threshold or comparatively broad are assigned to the second-lowest $J_{c}=11 / 2$ ionization threshold and are plotted against their corresponding principle quantum number (grey). Solid lines show the expected Rydberg energies using the simple Rydberg formula with the derived $E_{\mathrm{ion}, j}$.

observed Rydberg states as a function of their effective quantum numbers. We observe the typical $1 / n^{2}$ scaling of Rydberg states. We also found several very strong EIT features, at least ten times stronger than any surrounding resonances. Together with a few states located above the first ionization threshold, we assign them to be part of the Rydberg series attached to the second-lowest $J_{c}=11 / 2$ ionization threshold. Also, here we observe a similar $1 / n^{2}$ scaling which, together with their positions agreeing with the expected locations of states from this threshold, further strengthens our assignment.

\section{A. Determination of lowest ionization threshold and assignment of series}

As an important parameter for the assignment of quantum numbers and the understanding of the Rydberg series, we first determine the lowest ionization threshold by plotting the effective quantum numbers $v_{j}=\sqrt{R_{\mu} /\left(E_{\mathrm{ion}, j}-E_{i}\right)}$ and effective quantum defects $\mu_{j}=-v_{j}(\bmod 1)$. Here, $v_{j}$ is the effective quantum number with respect to the ion core state with angular momentum $j, E_{\text {ion }, j}$ the corresponding ionization threshold, and $E_{i}$ the energy of the Rydberg states. We also
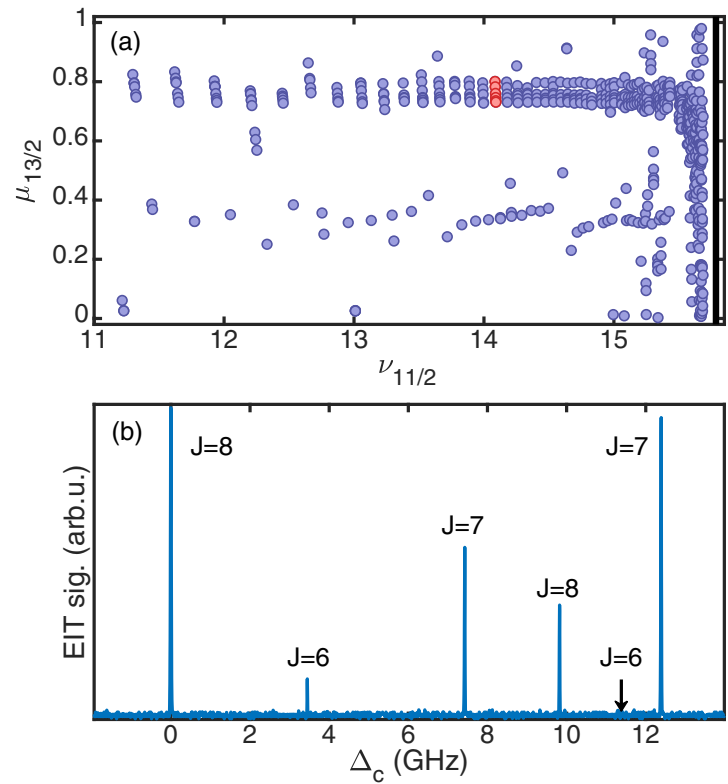

FIG. 3. Rydberg series: (a) Lu-Fano-style plot, showing the calculated $\mu_{13 / 2}$ of all observed states below the lowest ionization threshold as a function of the effective quantum number $v_{11 / 2}$ of the second-lowest ionization threshold. (b) Zoom into the EIT spectrum around $49148 \mathrm{~cm}^{-1}$, showing the states marked as red data points in (a). This bundle of resonances is identified as the $31 d$ Ry state where the fine structure splitting leads to six features with $J=6,7$, or 8 , see Sec. IV B for the assignment.

use $n_{\text {eff }}=$ floor $\left(v_{\mathrm{j}}\right)$ as the integer part for the assignment. We use a Lu-Fano analysis of our data to extract a new value for the ionization threshold: For an unperturbed Rydberg series, the quantum defect is nearly constant for intermediate principle quantum numbers. For Rydberg states with very high principle quantum numbers close to the ionization threshold, external influences like electric fields can disturb the states, and uncertainties in absolute frequencies have a larger influence on the effective quantum defect, while at lower energies the quantum defect shows a stronger state dependence. By plotting the calculated effective quantum defect $\mu_{13 / 2}$ versus $v_{11 / 2}$, we obtain a manifold of flat series around $\mu_{13 / 2}=0.8$. We vary the value for the ionization threshold and fit a straight line to all states with $0.7<\mu_{13 / 2}<0.9$ above $49250 \mathrm{~cm}^{-1}$ and find $E_{\text {ion, } 13 / 2}=49260.7442(23) \mathrm{cm}^{-1}$ as a value for the lowest ionization threshold to minimize the overall slope. This value is within the error margin of the literature value of $49262(8) \mathrm{cm}^{-1}$ [41] and of the preliminary value $49260.73(9) \mathrm{cm}^{-1}$ derived in Ref. [32], but improves in precision by almost four, or two, respectively, orders of magnitude. For the first excited ionization threshold, the same analysis suffers from the low number of states and missing states at high $v_{11 / 2}$. Therefore, we use the value reported in Refs. [42,43] for the splitting of the two states with $440.433(10) \mathrm{cm}^{-1}$ and calculate the $E_{\mathrm{ion}, 11 / 2}=$ 49701.177(10) $\mathrm{cm}^{-1}$.

Figure 3(a) shows the resulting effective quantum number and quantum defect for all states below the first ionization threshold. Two main series are visible, the first one around $\mu_{13 / 2}=0.35$ which consists of single separated states, 


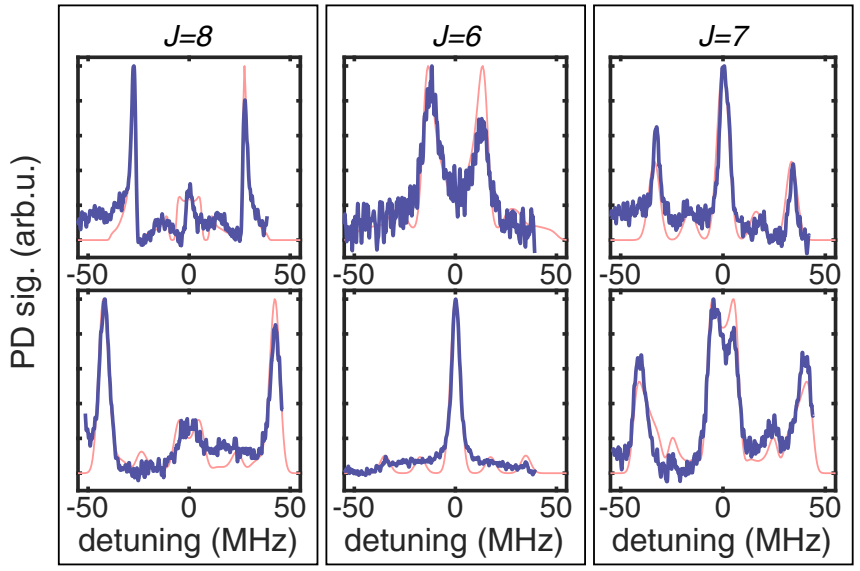

FIG. 4. Zeeman mapping of the $27 d$ fine structure multiplet. Blue thick lines show the photo diode signal of multiple averaged experimental data traces scanning the couple laser frequency. Red thin lines show the estimated spectral pattern using the fitted $g$ factors.

expected for the $n s$ series. At the second series around $\mu_{13 / 2}=$ 0.75 , we observe a bundle of five to six lines in relatively close proximity to each other. This is consistent with the expected $n d$ series. Figure 3(b) shows an exemplary scan over one of those bundles and already gives the assignment of the individual $J$ values, as we will detail below.

\section{B. Determination of total angular momentum $\boldsymbol{J}$ and $\boldsymbol{g}$ factors}

While the $n s$ states are clearly separated and are expected to be nearly exclusively $J=6$ (see Sec. V for a more detailed discussion), there is a large number of possible $n d$ states. A full assignment of the $n d$ states requires a determination of their total angular momentum, $J$, which we extract by performing a Zeeman spectroscopy for each state of the fine structure manifold for principal quantum numbers $n_{\mathrm{eff}}=$ 23, 27, 31, 37: We apply a magnetic field along $z$, and use horizontal polarization for all laser beams (propagating along $y$, polarization parallel to $x$ ), which provides $\sigma^{+}$and $\sigma^{-}$light in the reference frame of the atoms. We first calibrate our magnetic field with a Zeeman spectroscopy of the transition to the intermediate state without probe light by scanning the $401 \mathrm{~nm}$ laser and fitting Gaussian curves to the absorption signal. With the known $g$ factors for both ground and intermediate states, we determine our magnetic field strength to be 10.7(5) G.

Now we lock the probe beam frequency again on the zerofield resonance, scan the couple laser frequency over a range of about $100 \mathrm{MHz}$, and observe a splitting of the EIT lines, which results in distinctly different signal patterns, see exemplary the patterns of the $27 d$ fine-structure manifold shown in Fig. 4. We can sort the patterns into three groups: (A) One weak central peak and two strong peaks shifted symmetrically by about $40 \mathrm{MHz},(B)$ one central peak which may split into two peaks separated by less than $30 \mathrm{MHz}$, and (C) a strong central peak with two or four weaker side peaks. Note that the asymmetry in the spectra is an artifact from the measurement procedure which we were not able to remove completely.

These three distinct behaviors can be explained as the result of the combination of the specific $J$ in the ground $\left(J_{\mathrm{gs}}\right)$, excited $\left(J_{\mathrm{ex}}\right)$, and Rydberg states and the polarization of the probe and coupling beam. In general, the resonance frequency of a specific two-photon transition $\left|J_{\mathrm{gs}}, m_{\mathrm{gs}}\right\rangle \rightarrow\left|J, m_{J}\right\rangle$ shifts from its zero-field energy as $\Delta E=\left(g_{J} m_{J}-g_{\mathrm{gs}} m_{\mathrm{gs}}\right) \mu_{B} B$. Here, $m_{\mathrm{gs}}$ $\left(m_{J}\right)$ denotes the projection of $J_{\mathrm{gs}}(J)$ of the ground-state (Rydberg) atom along the quantization axis and $\Delta m=m_{J}-m_{\mathrm{gs}}$ the total difference between them. As transitions are limited to $|\Delta m| \leqslant 2$ due to selection rules and assuming that $g_{J}$ is close to $g_{\mathrm{gs}}$, we can identify five main features with energies:

$$
\begin{aligned}
\Delta E_{ \pm 2} & = \pm 2 g_{\mathrm{gs}} \mu_{B} B, \quad(\Delta m= \pm 2), \\
\Delta E_{ \pm 1} & = \pm 1 g_{\mathrm{gs}} \mu_{B} B, \quad(\Delta m= \pm 1), \\
\Delta E_{0} & =0, \quad\left(\Delta m_{J}=0\right) .
\end{aligned}
$$

Taking into account the additional difference in $g$ factors, each of these main resonances splits again into a series of closely spaced resonances with additional energy shifts $\Delta E=$ $\Delta g_{J} m_{J} \mu_{B} B$, with $\Delta g_{J}=g_{J}-g_{\mathrm{gs}}$.

Given our applied polarization of probe and coupling beam $\left(\sigma^{ \pm}\right)$and partial optical pumping toward the stretched states $J_{\mathrm{gs}}= \pm 6$ during the spectroscopy due to the different Clebsch-Gordan coefficients of the first transition, we expect mainly resonances at $\Delta E_{ \pm 2}$ and $\Delta E_{0}$, see Appendix $\mathrm{B}$ for further details and corresponding calculations. Based on these modeled spectral patterns, for $J=8$ we expect the strongest signals at $\Delta E_{ \pm 2}$ and a weaker central peak at $\Delta E_{0}$. Instead, for $J=6$, the strongest resonance will be $\Delta E_{0}$, which might split for large $\Delta g_{J}$, while $\Delta E_{ \pm 2}$ transitions will be very weak. Finally, $J=7$ will have its strongest component at $\Delta E_{0}$ together with slightly weaker $\Delta E_{ \pm 2}$ transitions and some very weak $\Delta E_{ \pm 1}$ components. With these considerations, we assign group (A) to $J=8$, group (B) to $J=6$, and group (C) to $J=7$.

Using this assignment technique for all investigated principal quantum numbers $n_{\text {eff }}=23,27,31,37$ [44], we observe the same fine-structure pattern with increasing energy, which goes as $J=8,6,7,8,6,7$, see Fig. 3(b). Additionally, we find that the $J=6$ EIT resonances within one scan are significantly weaker compared to $J=7,8$. The same pattern in relative signal strength can be found for five other $n_{\text {eff }}=$ $32-36$, allowing us to assign the total $J$ of each state via comparison of the order and relative height of the resonances of the EIT spectra without the need of a full Zeeman mapping for every $n_{\text {eff }}$.

We are also able to experimentally determine the $g_{J}$ value for most of the investigated states. For this, we fit our modeled spectral pattern (see Appendix B) to the experimental data, with $g_{J}$ as the main fitting parameter. We also allow some variation of the polarization and the optical pumping effect to be able to account for differences in the experimental conditions like probe and coupling laser intensities and beam alignment. Our results are summarized in Table IV.

\section{MQDT, ASSIGNMENT OF LINES, ASSIGNMENT OF QUANTUM DEFECTS}

Most of the experimentally measured energies for $20<$ $v_{13 / 2}<60$ seem to be grouped into two sets that are weakly interacting. The group with quantum defects $0.3 \lesssim \mu_{13 / 2} \lesssim$ 0.4 appear to come from a two channel series with one channel attached to the 13/2 threshold and the other attached to 
TABLE II. The MQDT parameters for the $n s J=6$ series.

\begin{tabular}{lcc}
\hline \hline$\tau$ & $\tau_{0}$ & $\tau_{2}$ \\
\hline$\mu_{1}$ & 0.30136 & -15.78 \\
$\mu_{2}$ & 0.42673 & -1.718 \\
$\alpha$ & 0.72378 & -0.594 \\
\hline \hline
\end{tabular}

the 11/2 threshold. From the discussion in Appendix A, this suggests that these are $J=6$ states with $n s$ Rydberg character. The other main group are states with quantum defects between $\sim 0.75$ and $\sim 0.8$ which, due to their number, must be states with $n d$ Rydberg character. There are several states with small quantum defects which might have $n g$ character. Finally, there are several states that are not part of either group but might be identified by fitting the MQDT parameters.

\section{A. $n s$ series}

In the limit that coupling between $n s$ and $n d$ states can be ignored, the $n s$ series with $J=6$ results from a two-channel system with one channel attached to the $J_{c}=13 / 2$ threshold and one attached to $J_{c}=11 / 2$. Since the $K$ matrix is symmetric there are only three independent parameters in the $K$ matrix. We chose the parameters to be the two eigenquantum defects and the mixing angle of the eigenvector. Taking channel 1 to be $J_{c}=13 / 2$ and 2 to be $J_{c}=11 / 2$, the $K$ matrix is written as

$$
K_{i j}=\sum_{a} U_{i a} U_{j a} \tan \left(\pi \mu_{a}\right),
$$

where $U_{11}=U_{22}=\cos (\alpha)$ and $U_{21}=-U_{12}=\sin (\alpha)$. The frame transformation approximation in Appendix A 3 implies $\alpha=\cos ^{-1}(\sqrt{7 / 13})=0.7469$.

We fit the identified states using these three parameters. The fit minimized the $\chi^{2}$ calculated from the sum of differences between the calculated and measured energies. When we take these parameters to be independent of energy, we find $\mu_{1}=0.27458, \mu_{2}=0.42223$, and $\alpha=0.72885$. Note the closeness of $\alpha$ to the value expected from the frame transformation approximation which further supports this approximation for the $n s$ series. The residuals of the $\mu_{13 / 2}$ are shown in Fig. 5(b) and for most of the states they are smaller than $\sim 0.01$. This level of agreement is roughly what should be expected for states over such a large range of energy. For a more realistic fit, we included a linear energy dependence in all of the parameters. Historical precedence gives a subscript 2 to the linear energy dependence [because the linear energy dependence was originally written as $\left.1 /(n-\mu)^{2}\right]$. For a parameter $\tau$, the energy dependence is written as

$$
\tau(E)=\tau_{0}+\eta(E) \tau_{2},
$$

where $\eta(E)=\left(E_{13 / 2}-E\right) / R_{166}$ and $R_{166}$ is the Rydberg constant defined in Appendix A. The fit values are given in Table II. Note that the value for $\alpha$ is only 3\% different from the frame transformation value. The comparison between the MQDT fit and measured bound state energies is shown in Fig. 5. Note that the residuals for all states are now much less than 0.01 . The fit was useful in identifying states not obviously part of the series with quantum defects between $\sim 0.3$

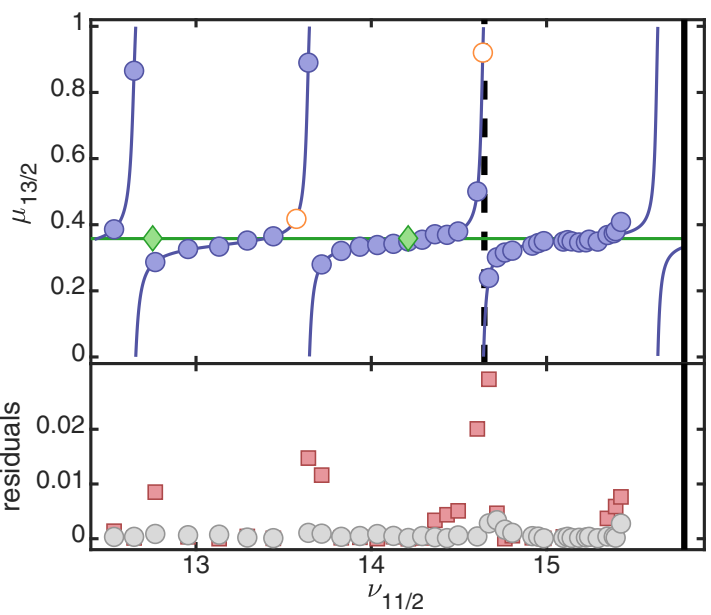

FIG. 5. Lu-Fano plot of the $s$ series states. (a) All experimentally observed states assigned to the $J=6$ (blue circles) and $J=7$ (green diamonds) $s$ series attached to the lowest $13 / 2$ ionization threshold. The lines show the result of the MQDT calculations with energy dependence for the $n s$ series; the black bar indicates the ionization threshold. The dashed line indicates a broad and strong EIT feature, assigned to the $n s$ series of the $11 / 2$ threshold. The open orange circles indicate theoretically predicted $J=6$ states found experimentally in an additional survey. (b) Absolute values of the difference between the measured data and the MQDT model, with (grey circles) and without (red squares) energy dependence (see Sec. V A for details on the model).

to $\sim 0.4$; these are the three states with quantum defects $\sim 0.9$. The fit also predicted two states not in the original data set that were subsequently identified: $26 s$ at $49105.45(7) \mathrm{cm}^{-1}$ $\left(\mu_{13 / 2} \simeq 0.42\right)$ and $39 \mathrm{~s}$ at $49188.91(7) \mathrm{cm}^{-1}\left(\mu_{13 / 2} \sim 0.92\right)$.

We observed only two states with quantum defects of $\mu=0.358$ that do not appear to be members of the $J=6$ series. We have assigned these to the $J=7$ series. This small number of observed states compared to $J=6$ might be explained by the different coupling of the two series. At short range, the $n s J=7$ must have the coupling $(6 s n s){ }^{3} \mathrm{~S}_{1}$. Since the intermediate state has $(6 s n p){ }^{1} \mathrm{P}_{1}$ character, transitions to the $J=7$ would be dipole suppressed. But since both the intermediate state and the Rydberg state have finite admixtures of triplet and singlet character, respectively, excitations are allowed, where the excitation strength strongly depends on the singlet admixture of the specific Rydberg state. The large difference of this quantum defect from the $\mu_{2}$ for $J=6$ suggests limitations to the frame transformation because these values should both be the ${ }^{3} \mathrm{~S}_{1}$ quantum defect, Appendix A 3 .

\section{B. $n d$ series}

The $n d$ series is more difficult to model because of the larger number of channels and the limited number of experimentally identified states being perturbed, see Sec. IV B above. In total, the experimental data allowed to identify 12 states with $J=6,18$ states with $J=7$, and 19 states with $J=8$ character. These states were used in the fit of the parameters. Because the $J=6$ and 7 have four channels and the $J=8$ has three channels, there are ten free parameters for $J=6$ and 7 and six free parameters for $J=8$. 
TABLE III. The MQDT parameters for the $n d$ series for different $J$.

\begin{tabular}{lcccc}
\hline \hline$J$ & $\mu\left({ }^{1} D_{2}\right)$ & $\mu\left({ }^{3} D_{1}\right)$ & $\mu\left({ }^{3} D_{2}\right)$ & $\mu\left({ }^{3} D_{3}\right)$ \\
\hline 6 & 0.782 & 0.729 & 0.744 & 0.791 \\
7 & 0.752 & 0.724 & 0.747 & 0.794 \\
8 & 0.750 & & 0.763 & 0.808 \\
\hline \hline
\end{tabular}

Symmetries in the bound-state conditions mean that the energies only determine eight parameters for $J=6$ and 7 and five parameters for $J=8$ (whenever two channels are attached to a threshold, the energies only determine the eigenquantum defects and cannot determine their mixing angle). The main difficulty in directly determining the full $K$ matrix is that the $J_{c}=13 / 2 n d$ states experimentally identified to a particular $J$ are not strongly perturbed by states attached to the $J_{c}=11 / 2$ threshold. This means that the eigenquantum defects attached to the $J_{c}=13 / 2$ threshold are well defined but most of the $K$-matrix elements are relatively unconstrained. As a contrast, see Fig. 5 where the perturbations of the $n s$ states clearly lead to variations greater than 0.1 for $\mu_{13 / 2}$. This means the energies are giving information about two parameters for each $J$.

These considerations suggest using the frame transformation approximation or interaction approximation, discussed in Appendices A 3 and A 4, respectively, to reduce the number of fit parameters. These approximations lead to nearly the same $\chi^{2}$ fit to the data points even though they have very different physical motivation. This is because they are mainly fitting to the eigenquantum defects of the channels attached to the $J_{c}=13 / 2$ threshold. For these fits, we did not include energy dependence in the MQDT parameters because the resulting fits did not substantially improve the agreement with experimental results.

In addition to the measured energies, we were able to experimentally determine the $g$ factor of several of the $n d$ states. Appendix A 2 describes an approximate method for calculating $g$ factors given the MQDT parameters. If the MQDT parameters were exact, this approximation would lead to errors less than $1 \%$. Unfortunately, we did not find an effective way of using the measured $g$ factors in the fitting procedure. The difference between the measured and simulated $g$ factors described below results from the limitations of the frame transformation and interaction approximations used in the $n d$ fits.

Figure 6 shows the experimental states where $J$ has been identified, together with the calculated nd series using the frame transformation approximation. This plot emphasizes that the fit gives a fairly accurate representation of the experimentally detected states. Table III shows the fit parameters for the frame transformation approximation. The fact that the quantum defects mostly do not vary strongly with $J$ (only the ${ }^{1} D_{2}$ varies by more than 0.02 ) suggests that this approximation captures much of the physics of these series. However, this approximation is not accurate enough to predict the states that are perturbed or are attached to the $J_{c}=11 / 2$ threshold, see Fig. 6. Also, the calculated $g$ factors substantially differ from the measured values, see Table IV. Both difficulties suggest that the frame transformation does not capture the

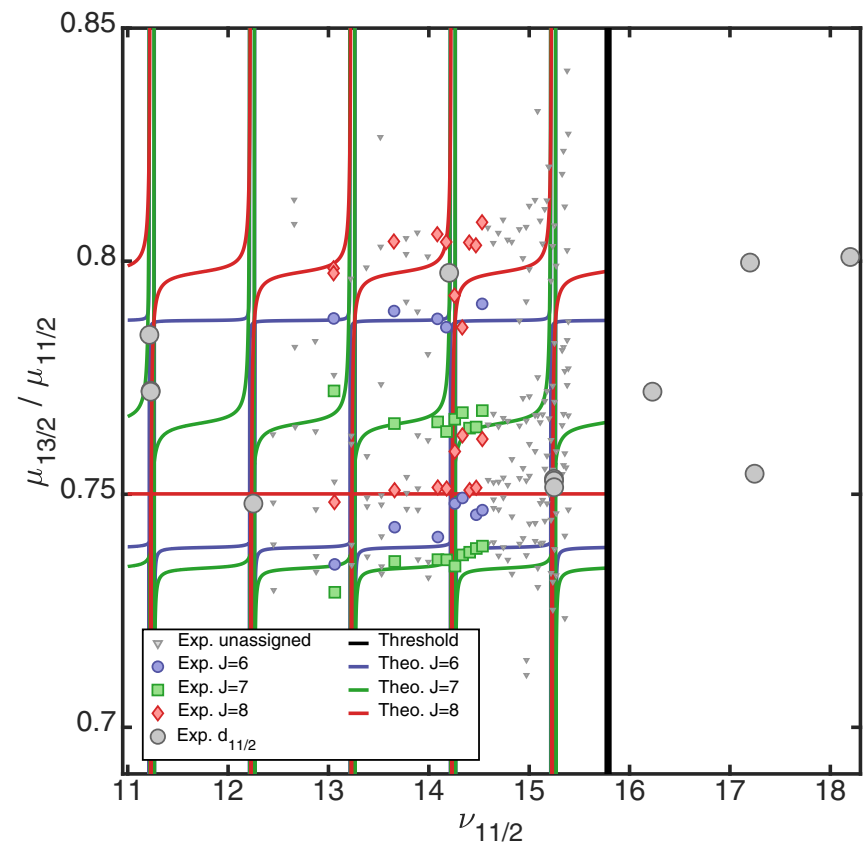

FIG. 6. MQDT assignment of $n d$ Rydberg series. The lines show the results of the frame transformation approximation fitting. Small symbols show all experimental data, colored filled symbols show all states where we assigned $J$ experimentally. Large grey circles (plotted with their respective $\mu_{11 / 2}$ ) represent states which we assign to the $J_{c}=11 / 2$ threshold. Four of these are above the first threshold, while states for four different $n$ are identified as perturbers of the $d$ series attached to the $J_{c}=13 / 2$ threshold.

full physics. The channel character is approximately 40:60 (or 60:40) of $\mathrm{nd}_{3 / 2}$ and $\mathrm{nd}_{5 / 2}$ for the frame transformation approximation. Thus, this approximation suggests the channels are strongly mixed. The fit to the interaction parameters, Appendix A 4, gives $C_{q}=0.7533, \mu_{3 / 2}=-0.0777, \mu_{6}=$ $0.7840, \mu_{7}=0.7690$, and $\mu_{8}=0.7979$. Since the experimental states are constraining six $K$-matrix parameters and there are five parameters in the fit, the fact that this model accurately reproduces the experimental values argues for the physics contained in the method. However, this approximation is also not accurate enough to predict the states that are perturbed or are attached to the $J_{c}=11 / 2$ threshold, see Fig. 6 . It does a better job of predicting the $g$ factor but still has substantial inaccuracies, see Table IV. The channel character is nearly pure for this approximation. However, the state designation is reversed from that used to experimentally determine the $g$ factor in Table IV. This contradicts the frame transformation characterization of the channel mixing which points to the uncertainty in the modeling of the $n d$ series.

Note that while the two-threshold MQDT analysis appears to predict much of the general pattern of the Rydberg levels and perturbations at least qualitatively correctly, the energy range just below the $J_{c}=13 / 2$ threshold looks irregular and even rather chaotic. To account for this apparent irregularity, it should be remembered that there are higher energy levels of $\mathrm{Er}^{+}$that are guaranteed to support their own infinite Rydberg series of levels as well. It is expected that some of the lowerlying members of those Rydberg series can also occur in the 
TABLE IV. List of fine-structure states with Zeeman mapping and measured $g$ factor, determined by energy splitting in a vertical magnetic field. For some states, the signal-to-noise ratio was too low to reliably fit the $g$ factor (-). The calculated $g$ factors are from the (frame transformation approximation, interaction fit). Note that the calculations only show a single state around 49056.9.

\begin{tabular}{lccccc}
\hline \hline$n_{\text {eff }}$ & $\mathrm{E}\left(\mathrm{cm}^{-1}\right)$ & $J$ & $g_{J, \text { meas }}$ & $g_{J, \text { diag }}$ & $g_{J, \text { calc }}(F T, Q P)$ \\
\hline 23 & $49056.886(5)$ & 8 & - & - & $(-,-)$ \\
23 & $49056.904(5)$ & 8 & $1.21(2)$ & 1.1493 & $(1.2205,1.2256)$ \\
23 & $49057.075(5)$ & 6 & $1.34(3)$ & 1.2454 & $(1.1597,1.2259)$ \\
23 & $49057.347(5)$ & 7 & $1.17(4)$ & 1.1877 & $(1.1491,1.2262)$ \\
23 & $49057.764(5)$ & 8 & - & 1.2234 & $(1.1520,1.1471)$ \\
23 & $49057.997(5)$ & 6 & $1.23(5)$ & 1.2293 & $(1.3148,1.2488)$ \\
23 & $49058.102(5)$ & 7 & $1.33(9)$ & 1.2258 & $(1.2640,1.1873)$ \\
27 & $49112.369(5)$ & 8 & $1.10(2)$ & 1.1493 & $(1.2206,1.2256)$ \\
27 & $49112.531(5)$ & 6 & $1.32(2)$ & 1.2454 & $(1.1597,1.2259)$ \\
27 & $49112.794(5)$ & 7 & $1.13(6)$ & 1.1877 & $(1.1492,1.2262)$ \\
27 & $49112.949(5)$ & 8 & $1.23(7)$ & 1.2234 & $(1.1520,1.1471)$ \\
27 & $49113.035(5)$ & 6 & $1.17(8)$ & 1.2293 & $(1.3149,1.2488)$ \\
27 & $49113.115(5)$ & 7 & $1.24(10)$ & 1.2258 & $(1.2642,1.1873)$ \\
31 & $49147.967(5)$ & 8 & $1.18(5)$ & 1.1493 & $(1.2206,1.2256)$ \\
31 & $49148.098(5)$ & 6 & $1.33(2)$ & 1.2454 & $(1.1597,1.2259)$ \\
31 & $49148.258(5)$ & 7 & $1.22(4)$ & 1.1877 & $(1.1491,1.2262)$ \\
31 & $49148.359(5)$ & 8 & $1.30(3)$ & 1.2234 & $(1.1520,1.1471)$ \\
31 & $49148.435(5)$ & 6 & $1.17(5)$ & 1.2293 & $(1.3149,1.2488)$ \\
31 & $49148.470(5)$ & 7 & $1.25(3)$ & 1.2258 & $(1.2641,1.1873)$ \\
37 & $49181.406(5)$ & 8 & $1.13(8)$ & 1.1493 & $(1.2207,1.2256)$ \\
37 & $49181.480(5)$ & 6 & - & 1.2454 & $(1.1597,1.2259)$ \\
37 & $49181.578(5)$ & 7 & $1.12(10)$ & 1.1877 & $(1.1492,1.2262)$ \\
37 & $49181.604(5)$ & 8 & $1.23(2)$ & 1.2234 & $(1.1520,1.1471)$ \\
37 & $49181.668(5)$ & 6 & - & 1.2293 & $(1.3149,1.2488)$ \\
37 & $49181.701(5)$ & 7 & $1.22(3)$ & 1.2258 & $(1.2642,1.1873)$ \\
\hline \hline & & & & & \\
\hline
\end{tabular}

spectral region studied here, and those are expected to cause significant distortions of the experimental energy level pattern and associated deviations from the present MQDT models.

One ionic threshold, in particular, appears to lie at an energy poised to produce such a perturbation just below the even-parity ground state of the ion. We refer to the odd-parity level of $\mathrm{Er}^{+}$with the spectroscopic label $4 f^{11} 6 s^{2}{ }^{4} \mathrm{I}_{15 / 2}^{o}$, which lies $6824.774 \mathrm{~cm}^{-1}$ above the $J_{c}=13 / 2$ ionic ground state. A $5 f$ Rydberg electron attached to that core, with an expected quantum defect of $\mu_{f} \approx 1$, should produce many Rydberg states of erbium lying approximately $34 \mathrm{~cm}^{-1}$ below the $J_{c}=$ $13 / 2$ ionization threshold, in the $v_{13 / 2} \approx 50-60$ range, and $v_{11 / 2} \approx 15.2$. Specifically, a $5 f_{5 / 2}$ electron produces levels with all values of $J$ from 5 to 10 , and a $5 f_{7 / 2}$ electron produces $J$ from 4 to 11 , so there are six perturbing levels expected near that region of the spectrum with angular momenta in the range $J=6-8$ that would be observable in the present experiment. Some of those levels might be present in Fig. 6, as that region near $v_{11 / 2} \approx 15.2$ shows numerous levels that deviate from our simplified two-threshold MQDT models.

\section{Coupling to low-lying $n g$ states}

Due to the angular momentum coupling to the ionic core, a two-photon excitation of a Rydberg state with $\ell=4$ ( $n g$ state) is possible as both angular momentum selection rules and

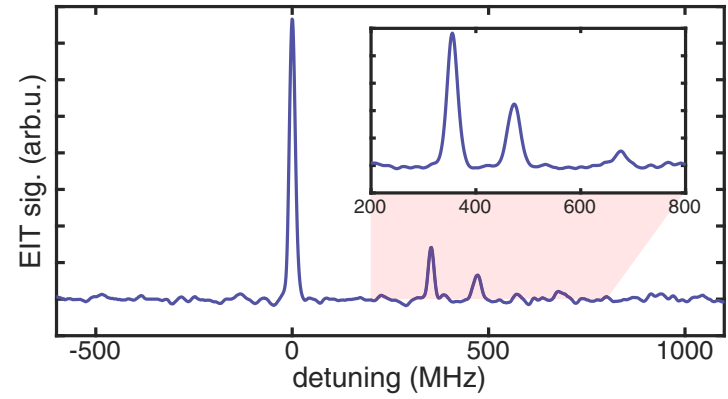

FIG. 7. EIT spectrum of the possible $13 g$ state at $49052.771 \mathrm{~cm}^{-1}$. A fine-structure splitting of four resonances is visible, the inset shows a second measurement run confirming the fourth resonance feature.

change of parity are fulfilled. Nonzero matrix elements can arise among others from $f$-wave admixture in the intermediate state or any $d$-wave admixture in the $n g$ Rydberg state. Below the first ionization threshold, the highest $n_{\mathrm{eff}, 11 / 2}=15$. Due to its large angular momentum, the corresponding quantum defect is expected to be close to zero. We identified several candidates with very small $\mu_{11 / 2}$ and further eliminated lines too close to other $n s$ or $n d$ resonances, especially when close to already identified perturbers. One candidate meets all requirements and additionally shows a distinct fine structure pattern different from the previous investigated series, see Fig. 7. Its energy of $49052.771 \mathrm{~cm}^{-1}$ would be compatible to a $13 \mathrm{~g}$ state connected to the second ionization threshold.

\section{States above first ionization threshold}

We observed several states above the first ionization threshold, which we assigned to the second ionization threshold. With an effective quantum defect $\mu_{11 / 2} \approx 0.78$, we would identify them as belonging to the $n d$ series of the $J=11 / 2$ state. We assign effective principal quantum numbers $n_{\text {eff, } 11 / 2}$ and measure their width as the FWHM value, as shown in Table V. Interestingly, their widths vary by more then two orders of magnitude, presumably caused by drastically different lifetimes. A more systematic survey of these states could improve the MQDT modeling by constraining the parameters, as discussed in Sec. V.

\section{E. Isotope shift}

To demonstrate the flexibility of our method and to exploit the large number of isotopes with high abundance in erbium,

TABLE V. States above the first ionization threshold, with their measured full width at half maximum. Only the first two states have significantly increased widths, while the other states show a linewidth comparable to the states below the threshold.

\begin{tabular}{lccc}
\hline \hline$n_{\text {eff, } 11 / 2}$ & $\delta_{\text {eff, } 11 / 2}$ & $E_{\text {meas }}\left(\mathrm{cm}^{-1}\right)$ & FWHM $(\mathrm{MHz})$ \\
\hline $17 d$ & 0.77 & $49284.43(1)$ & 350 \\
$18 d$ & 0.80 & $49330.22(1)$ & 30 \\
$18 d$ & 0.76 & $49332.16(1)$ & 2 \\
$19 d$ & 0.80 & $49369.82(1)$ & 4 \\
\hline \hline
\end{tabular}


TABLE VI. Total energy of the lowest state of the $27 d$ multiplets for different isotopes, with the energy difference between the Rydberg state and the intermediate $\left(E_{i, \mathrm{Ry}}\right)$ and the ground state $\left(E_{\mathrm{tot}}\right)$.

\begin{tabular}{lcc}
\hline \hline isotope & $E_{i, \mathrm{Ry}}\left(\mathrm{cm}^{-1}\right)$ & $E_{\text {tot }}\left(\mathrm{cm}^{-1}\right)$ \\
\hline 164 & $24169.102(5)$ & $49112.429(5)$ \\
166 & $24169.106(5)$ & $49112.404(5)$ \\
168 & $24169.105(5)$ & $49112.375(5)$ \\
170 & $24169.108(5)$ & $49112.350(5)$ \\
$167_{(17 / 2,19 / 2)}$ & $24169.031(5)$ & $49112.313(5)$ \\
$167_{(9 / 2,11 / 2)}$ & $24169.178(5)$ & $49112.465(5)$ \\
\hline \hline
\end{tabular}

we record the isotope shift of the $n=27 d$ multiplets for four bosonic isotopes, ${ }^{164} \mathrm{Er},{ }^{166} \mathrm{Er},{ }^{168} \mathrm{Er},{ }^{170} \mathrm{Er}$, as well as for two hyperfine states of the fermionic ${ }^{167} \mathrm{Er}$ isotope, see Table VI. This shows the versatility of our approach, and our ability to switch easily between the addressed isotopes and hyperfine states. For the energy of the first photon, we take the value from Ref. [45] to calculate the total energy.

\section{SUMMARY AND OUTLOOK}

We observe about 550 Rydberg states in erbium with unprecedented precision and with principal quantum numbers as high as $n=140$. By controlling the light polarization and applying a magnetic field, we can resolve the splitting between Zeeman sublevels and are able to assign the total angular momentum $J$ to the observed Rydberg series and measure their $g_{J}$ factors.

The number and precision of states allowed for an accurate determination of the MQDT parameters for the $n s$-Rydberg series. We were not able to unambiguously determine the MQDT parameters for the more complicated $n d$-Rydberg series. Two restricted models of the series were able to reproduce many of the features of the $n d$ series. However, the $g$ factors for several of the states and the details of the perturbations were not accurately reproduced. Future technical improvements will allow a higher absolute accuracy of the measured Rydberg energies as well as enabling a more systematic survey of the states between the two ionization thresholds, thus providing an improved basis for understanding and modeling of the $n d$-Rydberg series.

Our spectroscopic study marks a step in creating a toolbox for Rydberg physics. While our first survey concentrated on an excitation scheme using a $6 s$ electron, our vision is to employ schemes using inner-shell $4 f$ electrons. Additionally, to the possibilities offered by two-electron atoms like strontium or ytterbium, we expect dramatically new physics to be present and that the active submerged shell will affect fundamental properties of these systems.

\section{ACKNOWLEDGMENTS}

We thank Pascal Naubereith and Klaus Wendt for providing us unpublished spectroscopy data from the Mainz LARISSA project. This work is financially supported through an ERC Consolidator Grant (RARE, No. 681432). A.T. acknowledges support through the FWF Lise-Meitner Fellowship No. M 2683-N36. H.E. acknowledges support from the
Israel Council for Higher Education. F.R. received support from the U.S. Department of Energy, Office of Science, Basic Energy Sciences, under Award No. DE-SC0012193. C.H.G. received support from the U.S. Department of Energy, Office of Science, Basic Energy Sciences, under Award No. DESC0010545. We also acknowledge the Innsbruck Laser Core Facility, financed by the Austrian Federal Ministry of Science, Research and Economy. We acknowledge support by the Austrian Science Fund FWF within the DK-ALM (W1259-N27).

\section{APPENDIX A: MULTICHANNEL QUANTUM DEFECT EQUATIONS (MQDT)}

The formulas used to calculate the energies and $g$ factors of the states are given below. They follow the notation in Ref. [12] and are given with little discussion. For a fuller derivation and discussion, see Ref. [46] or [12]. The mass of ${ }^{166} \mathrm{Er}^{+}$was taken from Ref. [47] to be $M_{166+}=$ $165.9302931 u-m_{e}$ with $u=1.66053906660 \times 10^{-27} \mathrm{~kg}$ and $m_{e}=9.1093837015 \times 10^{-31} \mathrm{~kg}$ taken from the CODATA values. The Rydberg constant, $R_{166}$, was taken to be scaled from the CODATA $R_{\infty}$ value as $R_{166}=109737.315$ $68160 \mathrm{~cm}^{-1} \times M_{166+} /\left(M_{166+}+m_{e}\right)$.

\section{Calculated energies}

When the Rydberg electron is outside of the ionic core, the unphysical wave functions can be written as

$$
\left|\psi_{i}\right\rangle=\sum_{i^{\prime}}\left|\Phi_{i^{\prime}}\right\rangle\left[f_{i^{\prime}}(r) \delta_{i^{\prime}, i}-g_{i^{\prime}}(r) K_{i^{\prime}, i}\right],
$$

where the $f(g)$ are the energy normalized, radial Coulomb functions which are regular (irregular) at the origin and $\mathbf{K}$ is the real, symmetric $K$ matrix. See Ref. [46] for the properties of these functions that depend on the radial position of the Rydberg electron. The core states $\left|\Phi_{i^{\prime}}\right\rangle$ contain all other degrees of freedom. For the Rydberg states described above, the core states can be written as

$$
\left|\Phi_{i}\right\rangle=\left|\left(J_{c, i}\left(s \ell_{i}\right) j_{i}\right) J_{i} M_{i}\right\rangle,
$$

where $J_{c, i}$ is the total angular momentum of the core, $s$ is the spin of the Rydberg electron, $\ell_{i}$ is the orbital angular momentum of the Rydberg electron, $j_{i}$ is the total angular momentum of the Rydberg electron, $J_{i}$ is the total angular momentum, and $M_{i}$ is the related azimuthal quantum number. The order of parenthesis is meant to indicate the order that the angular momenta are coupled together.

The $\left|\psi_{i}\right\rangle$ function is unphysical because the $f_{i^{\prime}}, g_{i^{\prime}}$ functions diverge at large $r$ for closed channels defined by $E<$ $E_{c, i^{\prime}}$. At bound-state energies, the $\left|\psi_{i}\right\rangle$ can be superposed to give a physical eigenfunction which converges to 0 at large $r$. For the bound state at energy $E_{b}$, the superposition can be written as

$$
\left|\psi_{b}\right\rangle=\sum_{i}\left|\psi_{i}\right\rangle \frac{\cos \left(\beta_{i}\right)}{v_{i}^{3 / 2}} A_{i, b}
$$

and the condition that determines the bound states is [46]

$$
\sum_{i}\left[\tan \left(\beta_{i^{\prime}}\right) \delta_{i^{\prime}, i}+K_{i^{\prime}, i}\right] \frac{\cos \left(\beta_{i}\right)}{v_{i}^{3 / 2}} A_{i, b}=0,
$$


where $\beta_{i}=\pi\left(v_{i}-\ell_{i}\right)$ and the effective quantum number is defined as $E=E_{c, i}-R_{166} /\left(2 v_{i}^{2}\right)$ in terms of the total energy, $E$, and the energy of the $i$ th core state, $E_{c, i}$ with both energies in $\mathrm{cm}^{-1}$. The Rydberg constant is given in Appendix A above. This condition only holds when the term in [ ] has a determinant equal to 0 . The normalization condition when the $K$ matrix is slowly varying with energy is

$$
\sum_{i} A_{i, b}^{2}=1,
$$

which, with Eq. (A4), defines the $A_{i, b}$ within an irrelevant, overall sign.

\section{Calculated $g$ factor}

The $g$ factor for individual states can be approximately calculated from the MQDT parameters by assuming the contribution is negligible when the Rydberg electron is within the ionic core region. This situation is covered by Eq. (4.1.2) of Ref. [46] (or, similarly, Eqs. (15) and (16) of Ref. [12]):

$$
g_{b}=\sum_{i, i^{\prime}} A_{i, b}\left\langle\Phi_{i}|\hat{g}| \Phi_{i^{\prime}}\right\rangle O_{i b, i^{\prime} b} A_{i^{\prime} b},
$$

where the overlap matrix is

$$
O_{i b, i^{\prime} b}=\frac{2 \sqrt{\nu_{i b} v_{i^{\prime} b}}}{v_{i b}+v_{i^{\prime} b}} \frac{\sin \left(\beta_{i b}-\beta_{i^{\prime} b}\right)}{\left(\beta_{i b}-\beta_{i^{\prime} b}\right)},
$$

with $O_{i b, i b}=1$. The $g$-operator matrix element is complicated but only uses Eqs. (3.7.9), (5.4.1), (5.4.3), (7.1.7), and (7.1.8) of Ref. [48]. The matrix element is

$$
\begin{aligned}
\left\langle\Phi_{i}|\hat{g}| \Phi_{i^{\prime}}\right\rangle & =\frac{1}{M_{i}}\left\langle\Phi_{i}\left|g_{c, i} J_{c, z}+\ell_{z}+g_{s} s_{z}\right| \Phi_{i^{\prime}}\right\rangle \\
& =\frac{\left\langle\Phi_{i}|| g_{c, i} J_{c}^{(1)}+\ell^{(1)}+g_{s} s^{(1)} \| \Phi_{i^{\prime}}\right\rangle}{\Lambda\left(J_{i}\right)},
\end{aligned}
$$

where $g_{c}=1.230$ for the $J_{c}=13 / 2$ state and 1.101 for the $J_{c}=11 / 2$ state $[49,50]$ and $g_{s}=2.002319 \ldots$ The $\Lambda(x) \equiv$ $\sqrt{(2 x+1)(x+1) x}$. The reduced matrix elements when $\ell$ is the same for $i$ and $i^{\prime}$ are

$$
\begin{aligned}
\left\langle\Phi_{i}\left\|\ell^{(1)}\right\| \Phi_{i^{\prime}}\right\rangle & =\delta_{J_{c, i} J_{c, i^{\prime}}} \mathcal{G}_{1} \mathcal{G}_{2} \Lambda(\ell), \\
\left\langle\Phi_{i}\left\|s^{(1)}\right\| \Phi_{i^{\prime}}\right\rangle & =\delta_{J_{c, i} J_{c, i^{\prime}}} \mathcal{G}_{1} \mathcal{G}_{3} \Lambda(s), \\
\left\langle\Phi_{i}\left\|J_{c}^{(1)}\right\| \Phi_{i^{\prime}}\right\rangle & =\delta_{j_{i} j_{i^{\prime}}} \delta_{J_{c, i} J_{c, i^{\prime}}} \mathcal{G}_{4} \Lambda\left(J_{c, i}\right),
\end{aligned}
$$

where we have made the approximation that the core angular momentum operator does not mix core states with different $J_{c}$ and

$$
\begin{aligned}
& \mathcal{G}_{1}=(-1)^{J_{c, i}+j_{i^{\prime}}+J+1}(2 J+1)\left\{\begin{array}{ccc}
j_{i} & J & J_{c, i} \\
J & j_{i^{\prime}} & 1
\end{array}\right\}, \\
& \mathcal{G}_{2}=(-1)^{s+\ell+j_{i}+1}\left[j_{i}, j_{i^{\prime}}\right]\left\{\begin{array}{ccc}
\ell & j_{i} & s \\
j_{i^{\prime}} & \ell & 1
\end{array}\right\}, \\
& \mathcal{G}_{3}=(-1)^{s+\ell+j_{i^{\prime}}+1}\left[j_{i}, j_{i^{\prime}}\right]\left\{\begin{array}{ccc}
s & j_{i} & \ell \\
j_{i^{\prime}} & s & 1
\end{array}\right\}, \\
& \mathcal{G}_{4}=(-1)^{J_{c, i}+j_{i}+J+1}(2 J+1)\left\{\begin{array}{ccc}
J_{c, i} & J & j_{i} \\
J & J_{c, i^{\prime}} & 1
\end{array}\right\} .
\end{aligned}
$$

\section{Frame transformation approximation}

The energy-dependent $K$ matrix exactly determines the bound-state energies and approximately allows evaluation of other state properties (e.g., the $g$ factor). Unfortunately, the electronic structure of Er is too complicated for an accurate, $a b$ initio calculation. Nevertheless, there are two possible paths to obtain the $K$ matrix. The first is to use the experimental data, without any guidance from a model, to obtain a fit of the $K$ matrix. This is described in Sec. V A. The other is to utilize an approximation to restrict the possible values of the $K$ matrix before using the experimental energies to fit the elements of the $K$ matrix. This section describes this second method where the approximation is based on a frame transformation. The following section will use a different physical idea to reduce the number of parameters defining the $K$ matrix.

The frame transformation approximation assumes there is a channel coupling that diagonalizes the $K$ matrix when the electron is in the core region. The Rydberg states are attached to fine structure split core states of $\mathrm{Er}^{+}: 4 f^{12}\left({ }^{3} \mathrm{H}_{6}\right) 6 s_{1 / 2} J_{c}$ with $J_{c}=13 / 2$ and $11 / 2$ being the ground and first excited states, respectively. The idea is to call the angular momentum of the $4 f^{12}$ inner electrons $J_{f}=6$ and the angular momentum of the valence $6 s$ electron $J_{s}=1 / 2$. In terms of the notation of Appendix A 1, the channel states when the electron is outside of the core, Eq. (A2), is expanded to

$$
\left|\Phi_{i}\right\rangle=\left|\left(\left(J_{f} J_{s}\right) J_{c, i}\left(s \ell_{i}\right) j_{i}\right) J_{i} M_{i}\right\rangle
$$

We assume that the coupling that leads to a diagonal $K$ matrix is given by

$$
\left|\Phi_{i^{\prime}}^{i n}\right\rangle=\left|\left(J_{f}\left(\left(J_{s} s\right) S_{o, i^{\prime}} \ell_{i^{\prime}}\right) J_{o, i^{\prime}}\right) J_{i^{\prime}} M_{i^{\prime}}\right\rangle,
$$

where the order of coupling is the spin of the $6 s$ and Rydberg electron coupled to give total outer spin, $S_{o, i}$, then the total outer spin is coupled to the orbital angular momentum of the Rydberg electron, $\ell_{i}$, to give the total outer angular momentum, $J_{o, i}$, then the angular momentum of the inner $4 f^{12}$, $J_{f}=6$ is coupled to the total outer angular momentum to give the total angular momentum. The overlap matrix between these couplings are only nonzero if $J_{i}=J_{i^{\prime}}, M_{i}=M_{i^{\prime}}$, and $\ell_{i}=\ell_{i^{\prime}}$. For Er, the $s=1 / 2$ and is automatically the same.

With the help of the intermediate coupling $\left|\left(J_{f}\left(J_{s}(s \ell) j\right) J_{o}\right) J M\right\rangle$, the projection of the two coupling schemes is derived from Eq. (6.1.5) of Ref. [48] to give

$$
\begin{aligned}
\left\langle\Phi_{i} \mid \Phi_{i^{\prime}}^{i n}\right\rangle= & {\left[j_{i}, S_{o, i^{\prime}}, J_{c, i}, J_{o, i^{\prime}}\right](-1)^{J_{f}+2 J_{s}+j_{i}+J+s+\ell+J_{o, i^{\prime}}} } \\
& \times\left\{\begin{array}{ccc}
J_{f} & J_{s} & J_{c, i} \\
j_{i} & J & J_{o, i^{\prime}}
\end{array}\right\}\left\{\begin{array}{ccc}
J_{s} & s & S_{o, i^{\prime}} \\
\ell & J_{o, i^{\prime}} & j_{i}
\end{array}\right\},
\end{aligned}
$$

with $\left[j_{1}, j_{2} \ldots\right]=\sqrt{\left(2 j_{1}+1\right)\left(2 j_{2}+1\right) \ldots}$.

For the case where the Rydberg electron has $s$ character, there are two channels for $J=6$ and one channel for $J=7$. Thus, the frame transformation is only useful for $J=6$. The two $|\Phi\rangle$ states can be defined as $\left|J_{c}\right\rangle \equiv\left(\left({ }^{3} H_{6} 6 s_{1 / 2}\right) J_{c} n s_{1 / 2}\right) J=6$, with $J_{c}=13 / 2$ and $11 / 2$ while the two $\left|\Phi^{\text {in }}\right\rangle$ can be defined as $\left|J_{o}\right\rangle \equiv\left({ }^{3} H_{6}\left(6 s_{1 / 2} n s_{1 / 2}\right)^{2 S_{o}+1} S_{S_{o}}\right) J=6$ with $S_{o}=0$ and 1 . In 
this simplified notation, $\langle 13 / 2 \mid 0\rangle=\langle 11 / 2 \mid 1\rangle=\sqrt{7 / 13}$ and $\langle 13 / 2 \mid 1\rangle=-\langle 11 / 2 \mid 0\rangle=\sqrt{6 / 13}$.

For the case where the Rydberg electron has $d$ character, there are 4 channels for $J=6$ and 7 and three channels for $J=8$. The $|\Phi\rangle$ can be defined as $\left|J_{c}, j\right\rangle \equiv$ $\left(\left({ }^{3} H_{6} 6 s_{1 / 2}\right) J_{c} n d_{j}\right) J$. Both $J=6$ and 7 have the four couplings $|13 / 2,3 / 2\rangle,|13 / 2,5 / 2\rangle,|11 / 2,3 / 2\rangle$, and $|11 / 2,5 / 2\rangle$ while $J=8$ only has $|13 / 2,3 / 2\rangle,|13 / 2,5 / 2\rangle$, and $|11 / 2,5 / 2\rangle$. The $\left|\Phi^{\text {in }}\right\rangle$ can be defined as $\left|S_{o}, J_{o}\right\rangle \equiv\left({ }^{3} H_{6}\left(6 s_{1 / 2} n d_{1 / 2}\right)^{2 S_{o}+1} D_{J_{o}}\right) J$. Both $J=6$ and 7 have four couplings, $|0,2\rangle,|1,1\rangle,|1,2\rangle$, and $|1,3\rangle$ while $J=8$ only has $|0,2\rangle,|1,2\rangle$, and $|1,3\rangle$. If we let the quantum defects vary with $J$, then there are 11 free parameters for all $K$ matrices.

\section{Interaction-inspired $K$-matrix approximation}

A completely different method for parametrizing the $K$ matrix for the nd channels is to identify interactions that lead to coupling between channels or shifts in channels. This section discusses some of the important interactions.

There are two shifts that are expected. The first is the average $K$ matrix will depend on $J$. The second is the shift due to the spin-orbit interaction. These lead to terms of the form:

$$
K_{i i^{\prime}}^{(d)}=\delta_{i, i^{\prime}}\left[\tan \left(\pi \mu_{J}\right)+\delta_{j_{i}, 3 / 2} \tan \left(\pi \mu_{3 / 2}\right)\right],
$$

where we have put all of the spin-orbit shift into the $n d_{3 / 2}$ channels.

Another important interaction arises from a second rank coupling of the core state interacting with the $n d$ electron. This type of interaction can arise through a quadrupole moment of the ionic core or from an anisotropic polarizability of the core [51]. Other long-range interaction terms between the Rydberg electron and the anisotropic ionic core could be introduced, such as the vector hyperpolarizabity term, but the present level of theory has not yet taken such interactions into account $[52,53]$. The angular part of the matrix element arises from

$$
Q_{i i^{\prime}}=\left\langle\Phi_{i}\left|P_{2}\left(\hat{r}_{c} \cdot \hat{r}\right)\right| \Phi_{i^{\prime}}\right\rangle,
$$

where $\left|\Phi_{i}\right\rangle$ are from Eq. (A2) and the $P_{2}\left(\hat{r}_{c} \cdot \hat{r}\right)$ is the Legendre polynomial of the Rydberg electron dotted into core electrons. To evaluate this, we use the fact that the $4 f^{12}$ electrons are coupled as $S_{f}=1, L_{f}=5$, and $J_{f}=6$ before coupling to the $6 s$ electron to give $J_{c}$. Using Eqs. (5.4.6), (7.1.6), (7.1.7), and (7.1.8) of Ref. [48], we find $Q_{i i^{\prime}}=C q_{i i^{\prime}}$,

$$
\begin{aligned}
q_{i i^{\prime}}= & (-1)^{2 J_{c, i^{\prime}}+2 j_{i}+J} \frac{\left[j_{i}, j_{i^{\prime}}, J_{c, i}, J_{c, i^{\prime}}\right]}{[J]}\left\{\begin{array}{ccc}
\ell & j_{i} & s \\
j_{i^{\prime}} & \ell & 2
\end{array}\right\} \\
& \times\left\{\begin{array}{ccc}
J & j_{i} & J_{c, i} \\
2 & J_{c, i^{\prime}} & j_{i^{\prime}}
\end{array}\right\}\left\{\begin{array}{ccc}
J_{f} & J_{c, i} & J_{s} \\
J_{c, i^{\prime}} & J_{f} & 2
\end{array}\right\},
\end{aligned}
$$

when making the approximation $\ell_{i}=\ell_{i^{\prime}}$. Since the size of the second rank coupling is unknown, we add this to the $K$ matrix with a fitting parameter to obtain the total:

$$
K_{i i^{\prime}}=K_{i i^{\prime}}^{(d)}+C_{q} q_{i i^{\prime}}
$$
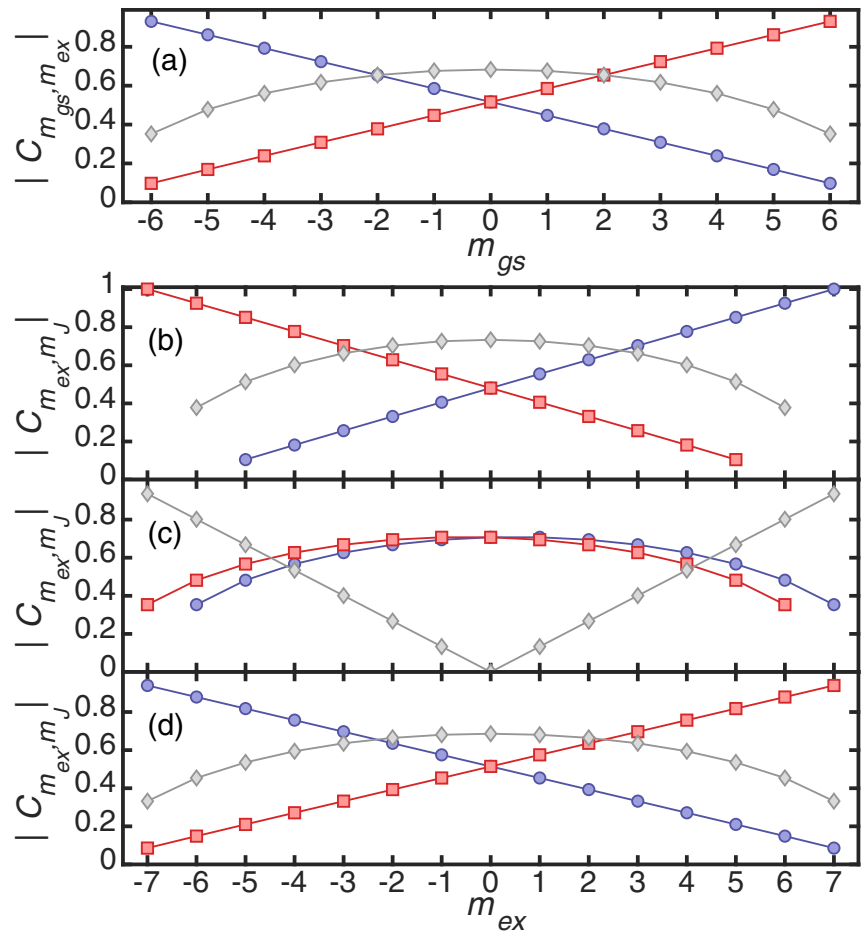

FIG. 8. Absolute values of the transition matrix elements for the first transition (a) $\left|J_{\mathrm{gs}}=6, m_{\mathrm{gs}}\right\rangle \rightarrow\left|J_{\mathrm{ex}}=7, m_{\mathrm{ex}}\right\rangle$ as well as for the three possibilities of the second transition, (b) $\left|J_{\mathrm{ex}}=7, m_{\mathrm{ex}}\right\rangle \rightarrow \mid J=$ $\left.6, m_{J}\right\rangle$, (c) $\left|J_{\mathrm{ex}}=7, m_{\mathrm{ex}}\right\rangle \rightarrow\left|J=7, m_{J}\right\rangle$, and (d) $\left|J_{\mathrm{ex}}=7, m_{\mathrm{ex}}\right\rangle \rightarrow$ $\left|J=8, m_{J}\right\rangle$. Circles (squares) encode $\sigma^{-}\left(\sigma^{+}\right)$transitions, diamonds display $\pi$ transitions.

If we stop at this level, there are five free parameters in the $K$ matrix.

\section{APPENDIX B: ESTIMATION OF THE SPECTRAL PATTERN OF DIFFERENT $J$ STATES OF THE $n d$ MANIFOLD}

For a simple estimation of the relative EIT resonance strength, we create a list of all dipole-allowed transitions from the ground state $\left|J_{\mathrm{gs}}=6, m_{\mathrm{gs}}\right\rangle$ over the intermediate state $\left|J_{\mathrm{ex}}=7, m_{\mathrm{ex}}\right\rangle$ to the Rydberg states $\left|J=6,7,8, m_{J}\right\rangle$. We calculate the transition matrix elements for each single photon transition using the Wigner $3 j$ symbol, leading to

$$
C_{m_{\mathrm{gs}}, m_{\mathrm{ex}}}=(-1)^{J_{\mathrm{gs}}-1+m_{\mathrm{ex}}} \sqrt{2 J_{\mathrm{gs}}+1}\left(\begin{array}{ccc}
J_{\mathrm{gs}} & 1 & J_{\mathrm{ex}} \\
m_{\mathrm{gs}} & \Delta m & -m_{\mathrm{ex}}
\end{array}\right)
$$

for the first transition and

$$
C_{m_{\mathrm{ex}}, m_{J}}=(-1)^{J_{\mathrm{ex}}-1+m_{J}} \sqrt{2 J_{\mathrm{ex}}+1}\left(\begin{array}{ccc}
J_{\mathrm{ex}} & 1 & J \\
m_{\mathrm{ex}} & \Delta m & -m_{J}
\end{array}\right)
$$

for the transition from the intermediate to the Rydberg state. Figure 8 shows the calculated values as a function of the $m_{J}$ state for all possible $J$ values.

For each possible combination, we can calculate the relative two-photon strength $\left|C_{m_{\mathrm{gs}}, m_{\mathrm{ex}}} \times C_{m_{\mathrm{ex}}, m_{J}}\right|$ and multiply it with our estimated amplitudes of our light polarizations (here we assume 5\% $\pi$-light) and the corresponding amplitude of 


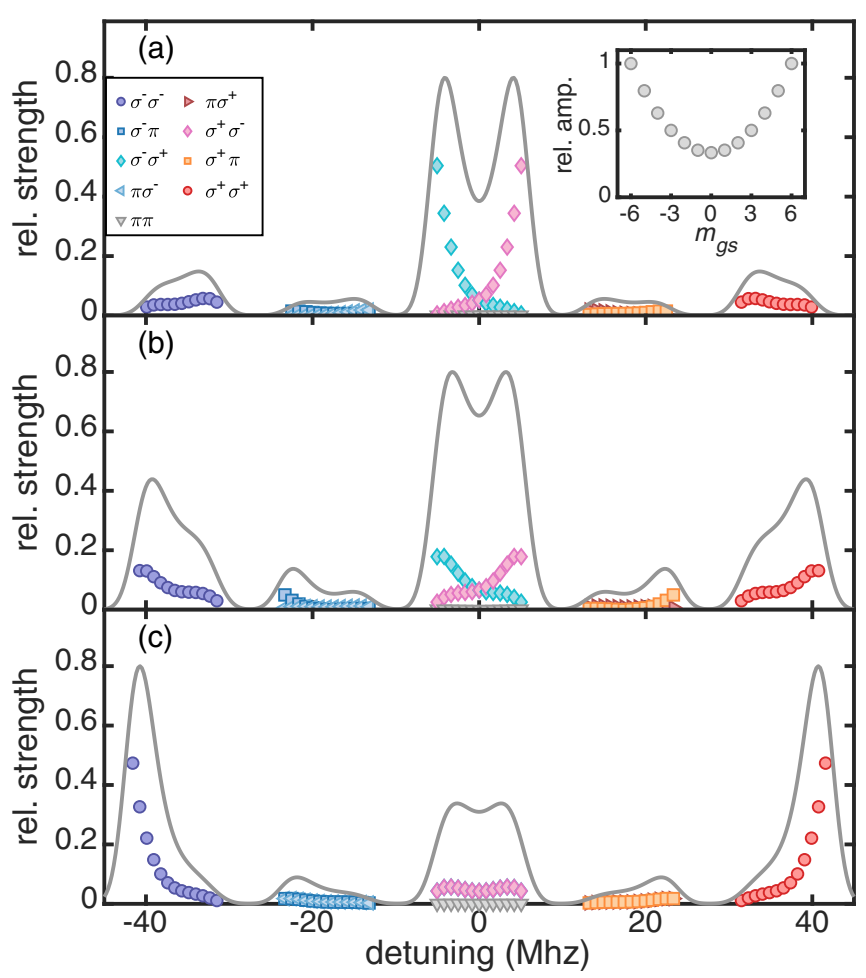

FIG. 9. Estimation of the spectral pattern given the relative twophoton transition strength for (a) $\left|J=6, m_{J}\right\rangle$, (b) $\left|J=7, m_{J}\right\rangle$, and (c) $\left|J=8, m_{J}\right\rangle$. Different colors and symbols encode the transition type (see legend). The inset shows the assumed relative distribution of populations among the ground-state manifold.

the population of the Zeeman level of the ground state. Here, the population amplitudes account for the effects of optical pumping during the spectroscopy. Now we can plot it as a function of the two-photon detuning for a given magnetic field, in our case $10.7 \mathrm{G}$, see Fig. 9. Here we exemplary assume a $g$ factor $g_{J}=1.22$ for the Rydberg state. The solid line gives a total sum of all transitions, taking a Gaussian line shape with a width of $2 \mathrm{MHz}$ and the corresponding two-photon strength as amplitude for each transition.

Each $J$ has its own characteristic spectral pattern: For $J=6$, a strong central peak originating from $\Delta m_{J}=0$ transitions (split for large $\left.g_{J}\right)$ with two weaker side peaks $\left(\Delta m_{J}=\right.$ \pm 2 transitions) is expected, while for $J=8$ the central peak should be much weaker and the side peaks should be strongest. For $J=7$, the central peak as well as the side peaks have roughly the same strength, and only here the $\Delta m_{J}= \pm 1$ transitions also have a significant strength and might be visible.

\section{APPENDIX C: ESTIMATION OF THE EXPERIMENTAL UNCERTAINTIES AND FREQUENCY INACCURACIES}

The used wave meter has an absolute frequency accuracy of $\pm 60 \mathrm{MHz}$, given as $3 \sigma$ interval [54]. This error is partially systematic, depending on various environmental conditions. Long-time measurements on a laser locked to an atomic tran- sition in our laboratory shows frequency deviations of typical $20 \mathrm{MHz}$ within one hour. We therefore assume a fundamental $1 \sigma$ error of $\pm 20 \mathrm{MHz}$ for single measurements and an additional $1 \sigma$ systematic uncertainty of $\pm 10 \mathrm{MHz}$ when combining multiple measurement sets.

To be able to give the absolute energy of the measured states, we have to add the first transition, whose energy we also measure with the same wave meter. We always measure the fundamental frequency of both probe and coupling beam before frequency doubling. We estimate our wavemeter-limited accuracy for single state energies therefore to about $\pm 69 \mathrm{MHz}$, i.e., $\pm 0.0023 \mathrm{~cm}^{-1}$. The final error in the determination of the ionization threshold includes the error of the fit itself and the combined systematic uncertainty of probe- and coupling frequency $\left( \pm 0.0013 \mathrm{~cm}^{-1}\right)$, resulting in an estimated accuracy of $\pm 0.0023 \mathrm{~cm}^{-1}$.

An additional uncertainty in our resonance position energies results from our wavelength monitoring. While scanning the coupling frequency using an analog voltage ramp, we continuously read out the measured frequencies from the wave meter. Due to the limited and variable read-out speed (depending on the light intensity coupled to the wave meter), which is not synchronized to the analog ramp, we record frequency traces during several scans and take the maximum and minimum measured wavelength to calibrate the frequency axis of the scans.

For most of the wide scans used in our survey, we estimate the absolute uncertainty of the resonance positions with $0.07 \mathrm{~cm}^{-1}$, mainly limited by nonlinearities within each frequency scan. For the nd states investigated for the $g$ factor, the smaller scan ranges minimize those nonlinearities and allows us to reduce the absolute uncertainty to $0.005 \mathrm{~cm}^{-1}$, now primarily limited by the absolute accuracy of the wave meter.

The main uncertainty in the fitting of the $g$ factor is the determination of the proper frequency axis scale. For these very narrow scan ranges, the finite read-out speed of the wave meter results in significant variations of the frequency axis scale of up to $20 \%$ when comparing different experimental runs where the scan range should have been identical. We account for this by scaling all frequency axes of one set to the maximum range, as the described frequency axis calibration will always lead to an underestimation of the scan range. The given error of the $g$ factor takes into account the fit error itself, the uncertainty of the $B$-field calibration and the rescaling factor.

\section{APPENDIX D: LIST OF ALL OBSERVED STATES}

All Rydberg features observed in this work using the EIT technique are summarized in Table VII. We provide the energies, as determined with the wavemeter, with an upper bound of the error of $0.07 \mathrm{~cm}^{-1}$. Some features have been measured with higher precision, see main text. The states are sorted by effective quantum number, as assigned to the lower $(J=$ $13 / 2)$ and upper $(J=11 / 2)$ ionization threshold. 
TABLE VII. Table listing all observed features, listed by effective quantum number $n_{\text {eff }}$, and term energy. Errors in energy are $0.07 \mathrm{~cm}^{-1}$. Some features have been measured with higher precision, see main text.

\begin{tabular}{|c|c|c|c|c|c|c|c|c|c|c|}
\hline \multicolumn{11}{|c|}{$n_{\mathrm{eff}, 13 / 2} E\left(\mathrm{~cm}^{-1}\right)$} \\
\hline 15 & 48828.76 & & & & & & & & & \\
\hline 16 & 48841.30 & 48842.77 & 48842.83 & 48843.43 & 48844.77 & 48845.23 & 48863.11 & 48863.98 & & \\
\hline 17 & 48888.33 & 48889.26 & 48889.80 & 48889.93 & 48891.47 & 48892.07 & 48892.73 & 48909.31 & 48909.33 & \\
\hline 18 & 48929.32 & 48929.99 & 48930.00 & 48930.01 & 48930.03 & 48930.87 & 48931.46 & 48931.71 & 48931.94 & 48945.19 \\
\hline 19 & 48963.98 & 48964.28 & 48964.32 & 48964.92 & 48964.98 & 48965.52 & 48968.26 & 48969.00 & 48979.36 & \\
\hline 20 & 48992.82 & 48993.07 & 48993.20 & 48993.44 & 48993.70 & 49002.52 & & & & \\
\hline 21 & 49015.08 & 49016.30 & 49016.42 & 49017.03 & 49017.42 & 49026.46 & 49028.00 & & & \\
\hline 22 & 49038.45 & 49038.83 & 49039.17 & 49039.37 & 49039.43 & 49047.31 & 49052.80 & 49052.81 & 49052.81 & 49052.82 \\
\hline 23 & 49056.91 & 49056.93 & 49057.10 & 49057.37 & 49057.79 & 49058.02 & 49058.13 & 49064.84 & & \\
\hline 24 & 49073.44 & 49073.97 & 49073.99 & 49074.33 & 49074.39 & 49074.79 & 49080.13 & 49081.41 & & \\
\hline 25 & 49087.98 & 49088.54 & 49088.66 & 49088.68 & 49088.83 & 49093.78 & & & & \\
\hline 26 & 49100.58 & 49100.86 & 49101.22 & 49101.51 & 49101.54 & 49101.62 & 49101.70 & 49101.72 & 49105.45 & \\
\hline 27 & 49111.45 & 49112.39 & 49112.56 & 49112.82 & 49112.98 & 49113.06 & 49113.14 & 49117.95 & & \\
\hline 28 & 49122.73 & 49122.76 & 49122.86 & 49123.23 & 49123.26 & 49123.29 & 49127.35 & & & \\
\hline 29 & 49132.01 & 49132.16 & 49132.39 & 49132.49 & 49132.53 & 49132.63 & 49136.08 & & & \\
\hline 30 & 49140.44 & 49140.60 & 49140.75 & 49140.96 & 49140.98 & 49144.04 & & & & \\
\hline 31 & 49147.99 & 49148.12 & 49148.28 & 49148.38 & 49148.46 & 49148.50 & 49151.25 & 49151.27 & & \\
\hline 32 & 49154.90 & 49155.02 & 49155.17 & 49155.25 & 49155.35 & 49157.12 & 49157.74 & 49157.82 & & \\
\hline 33 & 49160.85 & 49161.25 & 49161.41 & 49161.45 & 49161.52 & 49161.60 & 49163.83 & & & \\
\hline 34 & 49167.02 & 49167.12 & 49167.15 & 49167.22 & 49167.29 & 49169.25 & & & & \\
\hline 35 & 49172.18 & 49172.38 & 49172.45 & 49172.51 & 49174.33 & & & & & \\
\hline 36 & 49177.01 & 49177.19 & 49177.25 & 49177.28 & 49177.31 & 49178.94 & & & & \\
\hline 37 & 49181.43 & 49181.51 & 49181.60 & 49181.63 & 49181.69 & 49181.73 & & & & \\
\hline 38 & 49185.54 & 49185.70 & 49185.75 & 49185.80 & 49186.73 & & & & & \\
\hline 39 & 49188.90 & 49188.91 & 49189.34 & 49189.52 & 49189.54 & 49189.55 & 49189.57 & 49191.35 & & \\
\hline 40 & 49192.84 & 49192.98 & 49193.02 & 49193.04 & 49193.07 & 49194.52 & & & & \\
\hline 41 & 49196.09 & 49196.22 & 49196.26 & 49196.28 & 49196.31 & 49197.61 & & & & \\
\hline 42 & 49199.13 & 49199.25 & 49199.29 & 49199.33 & 49200.52 & & & & & \\
\hline 43 & 49201.95 & 49202.08 & 49202.11 & 49202.15 & & & & & & \\
\hline 45 & 49207.04 & 49207.08 & 49207.14 & 49207.16 & 49207.20 & 49208.14 & & & & \\
\hline 46 & 49209.35 & 49209.42 & 49209.45 & 49209.49 & 49210.35 & & & & & \\
\hline 47 & 49211.49 & 49211.53 & 49211.58 & 49211.61 & 49211.64 & 49211.69 & 49211.69 & 49212.43 & 49213.08 & \\
\hline 48 & 49213.51 & 49213.53 & 49213.59 & 49213.61 & 49213.62 & 49213.64 & 49214.30 & & & \\
\hline 50 & 49217.20 & 49217.28 & 49217.29 & 49217.32 & 49217.32 & 49218.53 & & & & \\
\hline 51 & 49218.85 & 49218.95 & 49218.96 & 49219.01 & 49219.02 & 49219.63 & & & & \\
\hline 52 & 49220.48 & 49220.55 & 49220.57 & 49220.59 & 49221.17 & & & & & \\
\hline 53 & 49221.99 & 49222.04 & 49222.07 & 49222.08 & 49222.64 & & & & & \\
\hline 54 & 49223.40 & 49223.45 & 49223.47 & 49223.49 & & & & & & \\
\hline 55 & 49224.73 & 49224.79 & 49224.81 & 49224.82 & 49225.34 & & & & & \\
\hline 56 & 49226.00 & 49226.00 & 49226.06 & 49226.08 & 49226.73 & & & & & \\
\hline 57 & 49227.23 & 49227.27 & 49227.29 & 49227.30 & 49227.75 & & & & & \\
\hline 58 & 49228.37 & 49228.40 & 49228.42 & 49228.45 & 49228.45 & 49228.83 & 49228.86 & 49229.11 & 49229.20 & \\
\hline 59 & 49229.45 & 49229.47 & 49229.49 & 49229.51 & 49229.52 & 49229.83 & 49229.87 & & & \\
\hline 60 & 49230.49 & 49230.53 & 49230.54 & 49230.55 & & & & & & \\
\hline 61 & 49231.29 & 49231.35 & 49231.38 & 49231.40 & 49231.89 & & & & & \\
\hline 62 & 49232.59 & 49232.64 & 49232.67 & 49232.67 & 49232.69 & & & & & \\
\hline 64 & 49234.13 & 49234.16 & 49234.17 & 49234.18 & 49234.60 & 49234.62 & 49234.62 & 49234.64 & 49234.76 & \\
\hline 65 & 49234.93 & 49234.97 & 49234.98 & 49234.99 & 49235.29 & & & & & \\
\hline 66 & 49235.72 & 49235.74 & 49235.76 & 49235.77 & 49235.78 & 49236.04 & 49236.10 & 49236.14 & 49236.17 & \\
\hline 67 & 49236.50 & & & & & & & & & \\
\hline 68 & 49237.05 & 49237.14 & 49237.17 & 49237.18 & 49237.19 & 49237.46 & & & & \\
\hline 69 & 49237.83 & 49237.86 & 49237.87 & 49237.87 & 49237.89 & 49238.13 & & & & \\
\hline 71 & 49239.10 & 49239.14 & 49239.15 & & & & & & & \\
\hline 72 & 49239.69 & 49239.70 & 49239.71 & 49239.71 & 49239.71 & 49239.72 & 49239.94 & & & \\
\hline 73 & 49240.26 & 49240.27 & 49240.28 & 49240.29 & & & & & & \\
\hline 82 & 49244.51 & 49244.53 & 49244.54 & 49244.55 & & & & & & \\
\hline 83 & 49244.90 & 49244.91 & 49244.92 & & & & & & & \\
\hline
\end{tabular}


TABLE VII. (Continued.)

\begin{tabular}{|c|c|c|c|c|c|c|c|c|}
\hline \multicolumn{9}{|c|}{$n_{\mathrm{eff}, 13 / 2} E\left(\mathrm{~cm}^{-1}\right)$} \\
\hline 84 & 49245.28 & 49245.28 & 49245.29 & 49245.31 & 49245.32 & & & \\
\hline 85 & 49245.64 & 49245.65 & 49245.66 & 49245.67 & 49245.68 & & & \\
\hline 86 & 49245.98 & 49245.98 & 49245.99 & 49246.00 & 49246.00 & & & \\
\hline 92 & 49247.86 & 49247.87 & & & & & & \\
\hline 93 & 49248.11 & 49248.13 & 49248.14 & 49248.16 & & & & \\
\hline 94 & 49248.39 & 49248.39 & 49248.41 & & & & & \\
\hline 95 & 49248.66 & 49248.67 & 49248.67 & 49248.68 & & & & \\
\hline 96 & 49248.90 & 49248.91 & 49248.92 & 49248.93 & & & & \\
\hline 97 & 49249.15 & 49249.15 & 49249.17 & 49249.17 & 49249.17 & 49249.19 & & \\
\hline 98 & 49249.38 & 49249.39 & 49249.45 & 49249.47 & & & & \\
\hline 99 & 49249.61 & 49249.62 & 49249.63 & 49249.65 & & & & \\
\hline 100 & 49249.85 & 49249.85 & 49249.86 & 49249.86 & 49249.93 & 49249.95 & & \\
\hline 101 & 49250.02 & 49250.03 & 49250.05 & 49250.06 & 49250.16 & 49250.18 & & \\
\hline 102 & 49250.24 & 49250.25 & 49250.25 & 49250.26 & & & & \\
\hline 103 & 49250.48 & 49250.49 & & & & & & \\
\hline 104 & 49250.65 & 49250.67 & & & & & & \\
\hline 105 & 49250.85 & 49250.86 & & & & & & \\
\hline 106 & 49251.05 & 49251.08 & & & & & & \\
\hline 107 & 49251.20 & 49251.21 & & & & & & \\
\hline 108 & 49251.43 & 49251.44 & & & & & & \\
\hline 109 & 49251.55 & 49251.57 & 49251.59 & & & & & \\
\hline 110 & 49251.73 & 49251.74 & 49251.76 & & & & & \\
\hline 111 & 49251.89 & 49251.90 & 49251.91 & 49251.91 & 49251.93 & 49251.93 & & \\
\hline 112 & 49252.04 & 49252.05 & 49252.07 & 49252.08 & & & & \\
\hline 113 & 49252.19 & 49252.19 & 49252.22 & 49252.23 & & & & \\
\hline 114 & 49252.34 & 49252.35 & 49252.36 & & & & & \\
\hline 115 & 49252.45 & 49252.46 & 49252.46 & 49252.47 & & & & \\
\hline 118 & 49252.92 & 49252.95 & 49252.96 & & & & & \\
\hline 119 & 49253.04 & 49253.08 & 49253.08 & & & & & \\
\hline 120 & 49253.16 & 49253.19 & 49253.20 & & & & & \\
\hline 121 & 49253.27 & 49253.30 & 49253.31 & & & & & \\
\hline 122 & 49253.37 & 49253.41 & 49253.42 & 49253.45 & 49253.46 & 49253.48 & & \\
\hline 123 & 49253.51 & 49253.60 & & & & & & \\
\hline 124 & 49253.65 & 49253.66 & & & & & & \\
\hline 125 & 49253.72 & 49253.76 & 49253.77 & 49253.83 & & & & \\
\hline 126 & 49253.87 & 49253.87 & 49253.93 & & & & & \\
\hline 127 & 49253.97 & 49253.97 & 49254.02 & 49254.04 & & & & \\
\hline 128 & 49254.09 & 49254.09 & 49254.15 & & & & & \\
\hline 129 & 49254.19 & 49254.19 & 49254.24 & & & & & \\
\hline 130 & 49254.28 & 49254.28 & 49254.29 & 49254.31 & 49254.33 & 49254.35 & & \\
\hline 131 & 49254.38 & 49254.38 & 49254.39 & 49254.40 & 49254.43 & & & \\
\hline 132 & 49254.44 & 49254.49 & 49254.49 & 49254.52 & 49254.54 & & & \\
\hline 133 & 49254.58 & 49254.62 & & & & & & \\
\hline 134 & 49254.66 & 49254.70 & 49254.71 & & & & & \\
\hline 135 & 49254.73 & 49254.74 & 49254.77 & 49254.77 & 49254.78 & 49254.78 & 49254.78 & 49254.79 \\
\hline 136 & 49254.85 & 49254.86 & 49254.89 & & & & & \\
\hline 137 & 49254.93 & 49254.97 & & & & & & \\
\hline 138 & 49255.01 & 49255.05 & & & & & & \\
\hline 139 & 49255.09 & 49255.13 & & & & & & \\
\hline 140 & 49255.15 & 49255.17 & & & & & & \\
\hline \multicolumn{9}{|c|}{$n_{\mathrm{eff}, 11 / 2} E\left(\mathrm{~cm}^{-1}\right)$} \\
\hline 11 & 48830.62 & 48830.66 & & & & & & \\
\hline 12 & 48970.09 & & & & & & & \\
\hline 14 & 49157.12 & 49189.47 & & & & & & \\
\hline 15 & 49219.46 & 49228.23 & 49229.09 & 49229.11 & & & & \\
\hline 16 & 49284.46 & & & & & & & \\
\hline 17 & 49330.24 & 49332.19 & & & & & & \\
\hline 18 & 49369.84 & & & & & & & \\
\hline
\end{tabular}


[1] D. Jaksch, J. I. Cirac, P. Zoller, S. L. Rolston, R. Côté, and M. D. Lukin, Fast Quantum Gates for Neutral Atoms, Phys. Rev. Lett. 85, 2208 (2000).

[2] G. K. Brennen, I. H. Deutsch, and P. S. Jessen, Entangling dipole-dipole interactions for quantum logic with neutral atoms, Phys. Rev. A 61, 062309 (2000).

[3] M. Saffman, T. G. Walker, and K. Mølmer, Quantum information with Rydberg atoms, Rev. Mod. Phys. 82, 2313 (2010).

[4] A. Browaeys and T. Lahaye, Many-body physics with individually controlled Rydberg atoms, Nat. Phys. 16, 132 (2020).

[5] D. Barredo, S. de Léséleuc, V. Lienhard, T. Lahaye, and A. Browaeys, An atom-by-atom assembler of defect-free arbitrary 2D atomic arrays, Science 354, 1021 (2016).

[6] H. Bernien, S. Schwartz, A. Keesling, H. Levine, A. Omran, H. Pichler, S. Choi, A. S. Zibrov, M. Endres, M. Greiner, V. Vuletić, and M. D. Lukin, Probing many-body dynamics on a 51-atom quantum simulator, Nature (London) 551, 579 (2017).

[7] D. Barredo, V. Lienhard, S. de Léséleuc, T. Lahaye, and A. Browaeys, Synthetic three-dimensional atomic structures assembled atom by atom, Nature (London) 561, 79 (2018).

[8] K.-N. Schymik, V. Lienhard, D. Barredo, P. Scholl, H. Williams, A. Browaeys, and T. Lahaye, Enhanced atom-byatom assembly of arbitrary tweezer arrays, Phys. Rev. A 102, 063107 (2020).

[9] M. Saffman and K. Mølmer, Scaling the neutral-atom Rydberg gate quantum computer by collective encoding in holmium atoms, Phys. Rev. A 78, 012336 (2008).

[10] R. Mukherjee, J. Millen, R. Nath, M. P. A. Jones, and T. Pohl, Many-body physics with alkaline-earth Rydberg lattices, J. Phys. B: At. Mol. Opt. Phys. 44, 184010 (2011).

[11] T. Topcu and A. Derevianko, Divalent rydberg atoms in optical lattices: Intensity landscape and magic trapping, Phys. Rev. A 89, 023411 (2014).

[12] F. Robicheaux, D. W. Booth, and M. Saffman, Theory of longrange interactions for Rydberg states attached to hyperfine-split cores, Phys. Rev. A 97, 022508 (2018).

[13] J. Wilson, S. Saskin, Y. Meng, S. Ma, R. Dilip, A. Burgers, and J. Thompson, Trapped arrays of alkaline earth rydberg atoms in optical tweezers, arXiv:1912.08754 [quant-ph].

[14] J. Millen, G. Lochead, and M. P. A. Jones, Two-Electron Excitation of an Interacting Cold Rydberg Gas, Phys. Rev. Lett. 105, 213004 (2010).

[15] A. D. Bounds, N. C. Jackson, R. K. Hanley, R. Faoro, E. M. Bridge, P. Huillery, and M. P. A. Jones, RydbergDressed Magneto-Optical Trap, Phys. Rev. Lett. 120, 183401 (2018).

[16] F. Camargo, R. Schmidt, J. D. Whalen, R. Ding, G. Woehl, S. Yoshida, J. Burgdörfer, F. B. Dunning, H. R. Sadeghpour, E. Demler, and T. C. Killian, Creation of Rydberg Polarons in a Bose Gas, Phys. Rev. Lett. 120, 083401 (2018).

[17] L. Couturier, I. Nosske, F. Hu, C. Tan, C. Qiao, Y. H. Jiang, P. Chen, and M. Weidemüller, Measurement of the strontium triplet rydberg series by depletion spectroscopy of ultracold atoms, Phys. Rev. A 99, 022503 (2019).

[18] H. Lehec, A. Zuliani, W. Maineult, E. Luc-Koenig, P. Pillet, P. Cheinet, F. Niyaz, and T. F. Gallagher, Laser and microwave spectroscopy of even-parity Rydberg states of neutral ytterbium and multichannel-quantum-defect-theory analysis, Phys. Rev. A 98, 062506 (2018).
[19] M. A. Norcia, A. W. Young, and A. M. Kaufman, Microscopic Control and Detection of Ultracold Strontium in OpticalTweezer Arrays, Phys. Rev. X 8, 041054 (2018).

[20] A. Cooper, J. P. Covey, I. S. Madjarov, S. G. Porsev, M. S. Safronova, and M. Endres, Alkaline-Earth Atoms in Optical Tweezers, Phys. Rev. X 8, 041055 (2018).

[21] S. Saskin, J. T. Wilson, B. Grinkemeyer, and J. D. Thompson, Narrow-Line Cooling and Imaging of Ytterbium Atoms in an Optical Tweezer Array, Phys. Rev. Lett. 122, 143002 (2019).

[22] I. S. Madjarov, J. P. Covey, A. L. Shaw, J. Choi, A. Kale, A. Cooper, H. Pichler, V. Schkolnik, J. R. Williams, and M. Endres, High-fidelity entanglement and detection of alkalineearth Rydberg atoms, Nat. Phys. 16, 857 (2020).

[23] J. J. McClelland and J. L. Hanssen, Laser Cooling Without Repumping: A Magneto-Optical Trap for Erbium Atoms, Phys. Rev. Lett. 96, 143005 (2006).

[24] B. Lev, M. Lu, and S. H. Youn, Laser cooling and trapping the most magnetic atom, dysprosium, in Frontiers in Optics 2010/Laser Science XXVI (Optical Society of America, Washington, DC, 2010), p. STuD4.

[25] A. Frisch, K. Aikawa, M. Mark, A. Rietzler, J. Schindler, E. Zupanič, R. Grimm, and F. Ferlaino, Narrow-line magnetooptical trap for erbium, Phys. Rev. A 85, 051401(R) (2012).

[26] J. Miao, J. Hostetter, G. Stratis, and M. Saffman, Magnetooptical trapping of holmium atoms, Phys. Rev. A 89, 041401(R) (2014).

[27] I. S. Cojocaru, S. V. Pyatchenkov, S. A. Snigirev, I. A. Luchnikov, E. S. Kalganova, G. A. Vishnyakova, D. N. Kublikova, V. S. Bushmakin, E. T. Davletov, V. V. Tsyganok, O. V. Belyaeva, A. Khoroshilov, V. N. Sorokin, D. D. Sukachev, and A. V. Akimov, Light-assisted collisions in ultracold Tm atoms, Phys. Rev. A 95, 012706 (2017).

[28] J. Hostetter, J. D. Pritchard, J. E. Lawler, and M. Saffman, Measurement of holmium Rydberg series through magneto-optical trap depletion spectroscopy, Phys. Rev. A 91, 012507 (2015).

[29] Z. Hai-jun, X. Xiang-yuan, H. Wen, L. Liang-quan, and C. Dieyan, Study of high-lying excited states of rare-earth element dy by laser resonance ionization spectroscopy, Acta Phys. Sin. (Overseas Edn) 1, 19 (1992).

[30] D. Studer, P. Dyrauf, P. Naubereit, R. Heinke, and K. Wendt, Resonance ionization spectroscopy in dysprosium, Hyperfine Interact. 238, 8 (2016).

[31] D. Studer, S. Heinitz, R. Heinke, P. Naubereit, R. Dressler, C. Guerrero, U. Köster, D. Schumann, and K. Wendt, Atomic transitions and the first ionization potential of promethium determined by laser spectroscopy, Phys. Rev. A 99, 062513 (2019).

[32] D. Studer, Resonanzionisationsspektroskopie hochliegender Zustnde in Dysprosium und Erbium zur Entwicklung effizienter Anregungsschemata und Bestimmung des ersten Ionisationspotentials, Master's thesis, Johannes Gutenberg Universität Mainz, 2015 (private communication).

[33] K.-J. Boller, A. Imamoğlu, and S. E. Harris, Observation of Electromagnetically Induced Transparency, Phys. Rev. Lett. 66, 2593 (1991).

[34] A. K. Mohapatra, T. R. Jackson, and C. S. Adams, Coherent Optical Detection of Highly Excited Rydberg States Using Electromagnetically Induced Transparency, Phys. Rev. Lett. 98, 113003 (2007).

[35] S. Mauger, J. Millen, and M. P. A. Jones, Spectroscopy of 
strontium rydberg states using electromagnetically induced transparency, J. Phys. B: At., Mol. Opt. Phys. 40, F319 (2007).

[36] J. B. Naber, A. Tauschinsky, H. B. van Linden van den Heuvell, and R. J. C. Spreeuw, Electromagnetically induced transparency with Rydberg atoms across the Breit-Rabi regime, SciPost Phys. 2, 015 (2017).

[37] K. T. Lu and U. Fano, Graphic analysis of perturbed Rydberg series, Phys. Rev. A 2, 81 (1970).

[38] C. L. Vaillant, M. P. A. Jones, and R. M. Potvliege, Multichannel quantum defect theory of strontium bound Rydberg states, J. Phys. B: At., Mol. Opt. Phys. 47, 155001 (2014).

[39] P. Ilzhöfer, G. Durastante, A. Patscheider, A. Trautmann, M. J. Mark, and F. Ferlaino, Two-species five-beam magneto-optical trap for erbium and dysprosium, Phys. Rev. A 97, 023633 (2018).

[40] S. H. Autler and C. H. Townes, Stark effect in rapidly varying fields, Phys. Rev. 100, 703 (1955).

[41] E. F. Worden, R. W. Solarz, J. A. Paisner, and J. G. Conway, First ionization potentials of lanthanides by laser spectroscopy, J. Opt. Soc. Am. 68, 52 (1978).

[42] W. C. Martin, R. Zalubas, and L. Hagan, Atomic energy levels - the rare-earth elements, Nat. Stand. Ref. Data Ser. NSRDS-NBS 60 (1978).

[43] J.-F. Wyart and J. E. Lawler, Theoretical interpretation and new energy levels in Er II, Phys. Scr. 79, 045301 (2009).

[44] For $n_{\text {eff }}=23$ we observe two $J=8$ features very close together, which is not yet understood.
[45] A. Frisch, K. Aikawa, M. Mark, F. Ferlaino, E. Berseneva, and S. Kotochigova, Hyperfine structure of laser-cooling transitions in fermionic erbium-167, Phys. Rev. A 88, 032508 (2013).

[46] M. Aymar, C. H. Greene, and E. Luc-Koenig, Multichannel Rydberg spectroscopy of complex atoms, Rev. Mod. Phys. 68, 1015 (1996).

[47] J. R. de Laeter, J. K. Böhlke, P. D. Bièvre, H. Hidaka, H. S. Peiser, K. J. R. Rosman, and P. D. P. Taylor, Atomic weights of the elements. Review 2000 (IUPAC Technical Report), Pure Appl. Chem. 75, 683 (2003).

[48] A. Edmonds, Angular Momentum in Quantum Mechanics, 2nd Edition (Princeton University Press, Princeton, NJ, 1974).

[49] A. Kramida, Yu. Ralchenko, J. Reader, and NIST ASD Team, NIST Atomic Spectra Database (Ver. 5.8) (online). https:// physics.nist.gov/asd [February 17, 2021] (National Institute of Standards and Technology, Gaithersburg, MD, 2020).

[50] J. R. McNally and K. L. V. Sluis, Preliminary classification of the spectrum of singly ionized erbium (Er II), J. Opt. Soc. Am. 49, 200 (1959).

[51] J. H. Becher, S. Baier, K. Aikawa, M. Lepers, J.-F. Wyart, O. Dulieu, and F. Ferlaino, Anisotropic polarizability of erbium atoms, Phys. Rev. A 97, 012509 (2018).

[52] W. Clark and C. H. Greene, Adventures of a Rydberg electron in an anisotropic world, Rev. Mod. Phys. 71, 821 (1999).

[53] S. Watanabe and C. H. Greene, Atomic polarizability in negative-ion photodetachment, Phys. Rev. A 22, 158 (1980).

[54] J. Schäfer, Toptica Photonics AG (private communication). 